\title{
Chancen-Risiko-Klassifizierung eines Portfolios aus staatlich geförderten Altersvorsorgeprodukten und Empfehlungen für die Kundenberatung
}

\author{
Franziska Diez $\cdot$ Roman Horsky $\cdot$ Ralf Korn
}

Angenommen: 8. Dezember 2021 / Online publiziert: 10. Januar 2022

(C) Der/die Autor(en) 2022

Zusammenfassung Um die Möglichkeiten der Nutzung der Chancen-Risiko Klassen (CRK) für staatlich geförderte Altersvorsorgeprodukte durch die Produktinformationsstelle Altersvorsorge gGmbH (PIA) bei der Kundenberatung zu maximieren, entwickeln wir ein Verfahren zur Bestimmung der CRK verschiedener Portfolios solcher Produkte, so dass die CRK des Portfolios nicht größer ist als die Risikopräferenz des zu beratenden Kunden. Dafür betrachten wir zum einen ein Portfolio aus zwei neu zu erwerbenden Produkten und zum anderen eines aus einem bereits beim Kunden vorhandenen Produkt und einem Neuprodukt. Wir untersuchen die Eigenschaften der verschiedenen Chancen- und Risikoparameter samt zugehöriger Abbildungen und zeigen, dass ein Diversifikationseffekt bei der Klassifizierung vorliegt. Aufbauend auf den Eigenschaften erhalten wir als Ergebnis, dass als Obergrenze der CRK des Portfolios die gemittelten Endvermögen heranzuziehen sind und übersetzen dies in Empfehlungen für die Kundenberatung.

Schlüsselwörter Chancen-Risiko-Klassifizierung · Portfolio-CRK ·

Diversifikationseffekt · Kundenberatung

Franziska Diez · Roman Horsky · Ralf Korn

Abteilung Finanzmathematik, Fraunhofer ITWM, Fraunhofer-Platz 1, 67663 Kaiserslauten,

Deutschland

Franziska Diez

E-Mail: franziska.diez@itwm.fraunhofer.de

Roman Horsky

E-Mail: roman.horsky@itwm.fraunhofer.de

Ralf Korn $(\bowtie)$

Fachbereich Mathematik, TU Kaiserslautern, 67653 Kaiserslautern, Deutschland

E-Mail: korn@mathematik.uni-kl.de 


\section{Chance-risk classification of a portfolio of state-subsidized pension products and recommendations for customer consulting}

Abstract To optimize the use of the chance-risk classes (CRC) of state-subsidized pension products assigned by Produktinformationsstelle Altersvorsorge gGmbH (PIA), we develop a procedure for determining the chance-risk class of different portfolios of pension products under the constraint that the portfolio CRC does not exceed the customer's risk preference. For this, we consider a portfolio consisting of two new products as well as one of an product already owned by the customer and an offered new one. We examine the properties of the various chance and risk parameters as well as their corresponding mappings and show that a diversification effect exists. Based on the properties, we conclude that the average final contract values have to be used to obtain the upper bound of the chance-risk class of a portfolio. Finally, we translate our results into recommendation for customer consultation.

\section{Einleitung}

In der Finanzindustrie besteht die Produktberatung in der Regel aus zwei Teilen: Die Risikopräferenz des Kunden wird durch einen Fragebogen ermittelt und in einer Risikoklasse zusammengefasst. Auf Basis dieser werden ihm entsprechende Produkte angeboten. Dazu werden die Anlageprodukte in verschiedene Anlageklassen eingeteilt. Dieses Verfahren soll sicherstellen, dass dem Kunden Produkte empfohlen werden, die für ihn geeignet sind und seiner Risikopräferenz entsprechen. In Deutschland ist dies zum Beispiel in $\$ 64$ Abs. 3 Wertpapierhandelsgesetz ${ }^{1}$ festgelegt.

Ähnliche Verfahren sind in der Versicherungswirtschaft bei der Beratung zu Altersvorsorgeprodukten zwar etabliert, beziehen aber keine sich bereits im Besitz der Kunden befindlichen Produkte ein. Aufgrund von Diversifikationseffekten kann jedoch die Gesamtbetrachtung des Kundenportfolios zu einer anderen CRK führen, als sich durch eine Einzelbetrachtung der Produkte und ihrer jeweiligen CRK ergeben würde. Hieraus können unterschiedliche Anlageempfehlungen resultieren.

Deshalb erweitern wir für staatlich geförderte Altersvorsorgeprodukte den oben beschriebenen Beratungsansatz um die Berücksichtigung des Kundenportfolios. Wir wählen diese Produktart, da hier eine einheitliche Klassifizierung durch die Produktinformationsstelle Altersvorsorge gGmbH (PIA) vorliegt, die bei anderen Finanzprodukten fehlt. Weiterhin ist die Klassifizierung gesetzlich vorgeschrieben und in der Altersvorsorge-Produktinformationsblattverordnung (AltvPIBV) geregelt. Demnach wird jedes staatlich geförderte Altersvorsorgeprodukt einer von fünf sogenannten Chancen-Risiko Klassen (CRK) zugeordnet. Basierend auf dieser Klassifizierung entwickeln wir ein Verfahren zur Bestimmung der CRK eines Portfolios. Mit diesem sind wir in der Lage, ...

\footnotetext{
1 Siehe https://www.gesetze-im-internet.de/wphg/_64.html (letzter Zugriff: 27.07.2020).
} 
... den maximalen Beitrag in ein neues Rentenversicherungsprodukt zu berechnen, so dass das Portfolio des Kunden keine größere CRK besitzt als seine Risikopräferenz.

... dem Kunden eine breitere Palette an Produkten anzubieten. Dies ist besonders in der Versicherungsbranche wichtig, in der jede Versicherungsgesellschaft deutlich weniger Produkte als im Bankensektor besitzt.

... frühere Fehlberatungen über das Portfolio zu kompensieren.

... neue Rentenversicherungsprodukte mit einer spezifischen CRK als Obergrenze zu entwickeln.

Dabei werden auch Schwächen der bisherigen gesetzlich geregelten Vorgaben aufgezeigt und Verbesserungsvorschläge ausgearbeitet. So ist die Kenntnis der CRK der einzelnen Produkte des Portfolios nicht ausreichend, um die CRK des Portfolios zu bestimmen. Hier kann es auf Portfolioebene zu Fehlberatungen kommen, die zu vermeiden sind. Laut AltvPIBV dürfen aber nur die CRK veröffentlicht werden.

Der Artikel richtet sich an Produktentwickler und Aktuare in Banken und Lebensversicherungsunternehmen, die Kenntnisse über die Klassifizierung staatlich geförderter Altersvorsorgeprodukte durch die PIA besitzen, und nicht direkt an die Kundenberater selbst. Hierauf gehen wir am Ende des Abschnitts nochmal detailliert ein.

Der Artikel besteht aus zwei Teilen. Zunächst beschreiben wir die Klassifizierung staatlich geförderter Altersvorsorgeprodukte durch die PIA. Hierfür werden produktindividuell jeweils 10.000 Endvermögen simuliert und deren Gesamtheit dann jeweils auf Kennzahlen für die Chancen und das Risiko abgebildet, anhand derer das Produkt in eine der CRK eingeordnet wird. Wir beschäftigen uns dann mit den Eigenschaften dieser Abbildungen und den resultierenden Kennzahlen. Hierauf aufbauend zeigen wir in Kap. 3 die Existenz eines Diversifikationseffekts und bestimmen die CRK eines Portfolios. Wir betrachten dabei sowohl ein Portfolio aus zwei neuen Produkten als auch eines aus einem bereits erworbenen und einem neuen Altersvorsorgeprodukt. Aus unseren Ergebnissen leiten wir Empfehlungen für die Kundenberatung her, die die Produktentwickler und Aktuare zur Entwicklung bestimmter Tools für die Kundenberater (z. B. Software oder Tabellen) und zur Schulung dieser nutzen können.

\section{Klassifizierung staatlich geförderter Altersvorsorgeprodukte}

Seit 2017 ist gesetzlich geregelt, dass jedes in Deutschland verkaufte staatlich geförderte Altersvorsorgeprodukt in eine von fünf CRK eingestuft sein muss und diese auf dem zugehörigen Produktinformationsblatt (PIB) ausgewiesen ist. Diese Klassifizierung wird von der PIA vergeben und basiert auf stochastischen Kapitalmarktsimulationen und der Auswertung der sich daraus ergebenden Verteilungen von Ansparvermögen der dazugehörenden Altersvorsorgeverträge. Eine detaillierte Beschreibung des verwendeten Kapitalmarktmodells sowie der zugehörigen Implementierung findet man z.B. in Korn und Wagner (2018), im Buch von Glau et al. (2018) oder in Abschn. 3.2 von Korn und Wagner (2019). 


\subsection{Der Klassifizierungsalgorithmus}

Die Klassifizierung hängt von der Verteilung des Vertragsguthaben des Produktes am Ende der Ansparphase (dem Endvermögen) des Musterkunden ab, der in $\$ 14$ AltvPIBV definiert ist. Für den Musterkunden werden Verträge mit unterschiedlichen Standardansparphasen $T$ von 12, 20, 30 und 40 Jahren Laufzeit und Prämien

$$
P_{T}= \begin{cases}100 € & \text { monatlich laufender Beitrag in }[0, \mathrm{~T}] \\ 1.200 \cdot T € & \text { Einmalbeitrag zu Beginn der Ansparphase }\end{cases}
$$

für laufende und einmalige Prämienzahlungen gesetzlich vorgeschrieben. Staatliche Boni sind in den Prämien beinhaltet. Die zweite Variante der Beitragszahlungen wird herangezogen, wenn nach $\S 14$ (1) Satz 2 AltvPIBV das Produkt nur gegen Einmalbeitrag verkauft wird. Per Verordnung sind für jede Ansparphase des Musterkunden 10.000 Szenarien der Entwicklung des Vertragsvermögens zu erstellen. Diese Szenarien basieren auf der Simulation der Entwicklung von Zinsen und Aktien, die wir im Folgenden als Kapitalmarktszenarien bezeichnen. Bei der Simulation der Entwicklung des Vertragsvermögens werden Kosten und produktindividuelle Eigenschaften, wie z. B. eine vorhandene Bruttobeitragsgarantie oder eine konkrete statische oder dynamische Anlagestrategie der Beiträge des Kunden, berücksichtigt, während Managemententscheidungen, wie z.B. i.a. die nicht publik gemachte mittelfristige strategische Assetallokation des Unternehmens, nicht simuliert werden können. Die simulierten Endvermögen werden mit $v^{k} \geq 0, \mathrm{k}=1, \ldots, 10.000$ bezeichnet. Besitzt das Produkt eine vertraglich vereinbarte Bruttobeitragsgarantie, dann ist $v^{k}$ das Maximum aus den simulierten Endvermögen und 1.200 $T$.

Auf der Grundlage der Endvermögen $v^{k}$ werden das Chancen- und das Risikomaß berechnet, die als zwei konstante jährliche Zinssätze gegeben sind. Als Ausgangspunkt für das Chancenmaß wird der Mittelwert der simulierten Endvermögen berechnet

$$
{ }^{-c}=\mathbb{E}(V),
$$

wobei $\mathbb{E}$ den Erwartungswert in Bezug auf die empirische Verteilung der Stichprobe bezeichnet und mit $V$ das Altersvorsorgeprodukt bezeichnet wird, das über seine Endvermögen definiert ist. Das Risikomaß basiert auf dem Mittelwert der 2000 niedrigsten Endvermögen. Dieser ist gegeben durch

$$
{ }^{-r}=\mathbb{E}\left(V \mid V \leq Q_{0,2}\right),
$$

wobei $Q_{0,2}$ das $20 \%$-Quantil von $V$ ist.

Ausgehend von diesen Größen werden die konstanten jährlichen Zinssätze $\mu^{c}$ und $\mu^{r}$ auf den monatlichen Beitrag oder den Einmalbeitrag des Musterkunden ermittelt, die zu den oben berechneten Mittelwerten $V$ und $V$ führen. Hier wird ein deter- 
ministischer Sparprozess ohne Kosten angenommen. Der entsprechende Zinssatz $\mu$ berechnet sich dann bei gemitteltem Endvermögen $\bar{V}$ aus

$$
\bar{V}= \begin{cases}100 \sum_{j=1}^{12 T}\left(1+\frac{\mu}{12}\right)^{j} & \text { monatlich laufender Beitrag } \\ 1.200 \cdot T\left(1+\frac{\mu}{12}\right)^{12 T} & \text { Einmalbeitrag. }\end{cases}
$$

$\mu^{c}$ ist der Wert, der die Gl. 3 für $\bar{V}=\bar{V}^{c}$ löst, und $\mu^{r}$ der Wert, der die Gleichung für $\bar{V}=\bar{V}^{r}$ löst. Für $\mu \geq-12$ sind die jeweiligen Lösungen eindeutig, da dort die jeweiligen rechten Seiten der Gl. 3 streng monoton in $\mu$ wachsen und das vollständige Intervall $[0, \infty)$ als Wertebereich besitzen. Werte $\mu<-12$ sind ökonomisch irrelevant und werden hier nicht betrachtet.

Die beiden Kennzahlen $\mu^{c}$ und $\mu^{r}$ spannen eine Werteraum auf, der mit Hilfe von Referenzportfolien in fünf Bereiche eingeteilt wird (siehe Abschn. 2.2). Jedem Bereich wird eine CRK zugeordnet. Einem Altersvorsorgeprodukt wird dann die CRK des Bereichs zugewiesen, in den das Paar $\left(\mu^{c}, \mu^{r}\right)$ seiner ermittelten Chancenund Risikokennzahlen fällt.

Für jede Ansparphase des Musterkunden in Abhängigkeit von der Beitragzahlungsweise ermittelt die PIA für den Anbieter eine CRK. Nach §5 (2) Satz 4 AltvPIBV bestimmt die vereinbarte Ansparphase $T^{a}$ des Produktes die Ansparphase $T$ der Klassifizierung und damit, welche der vier CRK dem Produkt zuzuordnen ist. Es gilt, dass

$$
\text { für }\left\{\begin{array}{l}
T^{a} \leq 12 \\
12<T^{a} \leq 20 \\
20<T^{a} \leq 30 \\
T^{a}>30
\end{array}\right\} \text { die CRK, die für }\left\{\begin{array}{l}
T=12 \\
T=20 \\
T=30 \\
T=40
\end{array}\right\} \text { ermittelt wurde, }
$$

auf dem PIB auszuweisen ist.

\subsection{Chancen-Risiko Klassengrenzen}

„Die CRK gibt an, wie die Ertragschancen und Risiken dieses Produktes [...] einzuschätzen sind“"2, wobei eine höhere CRK mit höheren Risiken, aber auch mit höheren Ertragschancen einhergeht. Die CRK sind gesetzlich festgelegt, siehe Bundesministerium der Finanzen (2019) oder Produktinformationsstelle Altersvorsorge (2016), und wie folgt definiert:

CRK 1 Das Produkt bietet eine sichere Anlage durch eine bis zum Beginn der Auszahlungsphase festgelegte garantierte (Mindest-) Verzinsung oder an einen Referenzzins gekoppelte Verzinsung mit niedrigen Ertragschancen. Das unwiderruflich gebildete Kapital nach Abzug der Kosten steigt in der Ansparphase fortwährend an. Der Anbieter gibt eine Beitragserhaltungszusage.

\footnotetext{
2 Bundesministerium der Finanzen, S. 9.
} 
CRK 2 Das Produkt bietet eine sicherheitsorientierte Anlage mit begrenzten Ertragschancen. Der Anbieter gibt eine Beitragserhaltungszusage.

CRK 3 Das Produkt bietet eine ausgewogene Anlage mit moderaten Ertragschancen. Gibt der Anbieter keine Beitragserhaltungszusage, so besteht ein moderates Verlustrisiko.

CRK 4 Das Produkt bietet eine renditeorientierte Anlage mit höheren Ertragschancen. Gibt der Anbieter keine Beitragserhaltungszusage, so besteht ein höheres Verlustrisiko.

CRK 5 Das Produkt bietet eine chancenorientierte Anlage mit hohen Ertragschancen. Gibt der Anbieter keine Beitragserhaltungszusage, so besteht ein hohes Verlustrisiko.

Für die Einordnung eines Altersvorsorgeproduktes in eine CRK müssen die Klassen sowohl nach dem Chancen- als auch dem Risikomaß klar definiert sein. Um die Grenzen der CRK zu bestimmen, werden verschiedene Referenzportfolios simuliert. Diese besitzen keine Kosten und sind auf Basis von Nullkuponanleihen und einer riskanten Anlage definiert. Damit ist die Ermittlung der Klassengrenzen unabhängig von Kosten und den verschiedenen am Markt angebotenen Produktarten. Weiterhin sind die Referenzportfolios so gewählt, dass sie die Charakteristik der jeweiligen CRK repräsentieren. Sie sind wie folgt definiert (siehe Korn und Wagner 2019):

Referenzportfolio 1 Von jedem Beitrag werden Nullkuponanleihen mit Fälligkeit gleich dem Ende der Ansparphase gekauft.

Referenzportfolio 2 Von jedem Beitrag werden zum einen Nullkuponanleihen mit Fälligkeit gleich dem Ende der Ansparphase und Nominalwert gleich dem Beitrag (Absicherung des Beitrages) gekauft, zum anderen werden von dem verbleibenden Beitrag Call-Optionen auf die riskante Anlage mit einjähriger Laufzeit gekauft. Etwaige Gewinne aus den Optionen werden ebenfalls mit Hilfe von Nullkuponanleihen zum Laufzeitende gesichert.

Referenzportfolio $350 \%$ jedes Beitrages wird in 10-jährige Nullkuponanleihen investiert und der verbleibende Anteil in die riskante Anlage.

Referenzportfolio $425 \%$ jedes Beitrages wird in 10-jährige Nullkuponanleihen investiert und der verbleibende Anteil in die riskante Anlage.

Referenzportfolio 5 Der gesamte Beitrag wird in die riskante Anlage investiert. ${ }^{3}$ Für jedes Referenzportfolio werden das Chancen- und das Risikomaß wie in Abschn. 2.1 erläutert berechnet. Die Grenzen zwischen den CRK sind als eine Gerade mit Steigung 1 in einem $\mu^{c}-\mu^{r}$-Diagramm definiert. Mit Ausnahme der Klas-

\footnotetext{
3 Aufgrund der gegenwärtigen Zinslage würde mit dem Vorgehen in Referenzportfolio 1 und 2 nicht immer sichergestellt, dass die eingezahlte Beitragssumme zum Ende der Ansparphase garantiert werden kann.
} 
Abb. 1 Referenzportfolios und CRK-Grenzen

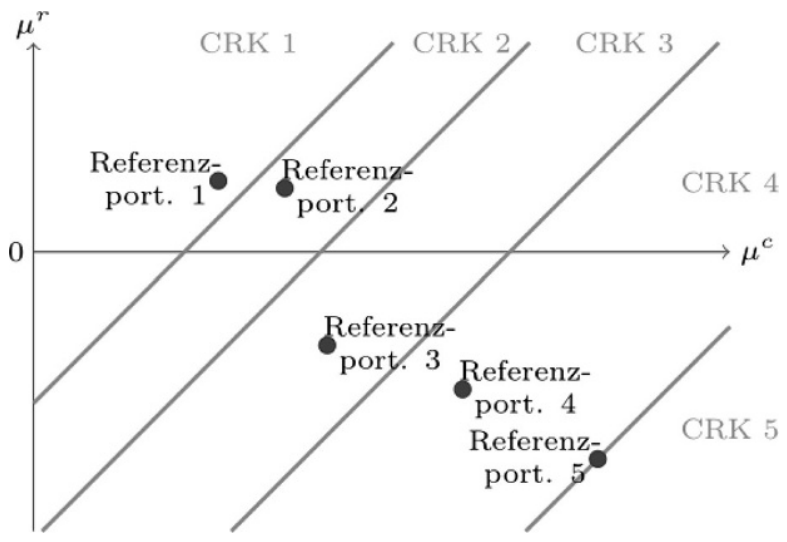

sengrenze zwischen CRK 4 und 5, die auf dem fünften Referenzportfolio liegt, durchläuft die Gerade den Mittelpunkt der linearen Verbindung zweier benachbarter Referenzportfolios. Für die verschiedenen Kombinationen aus Ansparphasen $T$ und Beitragszahlungsweisen ergeben sich unterschiedliche CRK-Grenzen. Abb. 1 veranschaulicht die CRK-Grenzen für eine solche Kombination.

Durch die Wahl der Steigung 1 der CRK-Grenzen kann jede Klassengrenze eindeutig durch einen Punkt auf der Geraden spezifiziert werden. Wir wählen hierfür den $\mu^{c}$-Achsenabschnitt, der das Chancenmaß der CRK-Grenzen bei einem Risikomaß von null darstellt. Diesen bezeichnen wir für die Grenze zwischen CRK $j$ und $j+1, j=1,2,3,4$, für die Ansparphase $T$ im Folgenden mit $b_{T}^{j}$.

\subsection{Eigenschaften der Chancen- und Risikomaße}

In diesem Abschnitt untersuchen wir die Abbildung der simulierten Endvermögen auf die gemittelten Endvermögen und dann weiter auf das Chancen- und das Risikomaß, sowie diese beiden Maße an sich auf ihre Eigenschaften. $\bar{V}^{r}$ und $\mu^{r}$, genauso wie $\bar{V}^{c}$ und $\mu^{c}$ quantifizieren den Ertrag eines Produktes und nicht den Verlust, wie es bei klassischen Risikomaßen üblich ist. Wir behalten die Bezeichnung Risikomaß bei, da dies die Bezeichnung auf dem PIB ist.

Da für alle Endvermögen $v^{k} \geq 0$ gilt, folgen $\bar{V}^{c} \geq 0$ und $\bar{V}^{r} \geq 0$ und somit auch $\mu^{c} \geq-12$ und $\mu^{r} \geq-12$. Weiterhin ist $\bar{V}^{c} \geq \bar{V}^{r}$ und $\mu^{c} \geq \mu^{r}$. Die Chancen- und Risikoparameter sind nur gleich, wenn die Endvermögen $v^{k}$ in allen 10.000 Simulationen gleich sind. Ein größerer Risikoparameter $\bar{V}^{r}$ oder $\mu^{r}$ ist immer besser als ein niedriger, da dieser Risikoparameter das Endvermögen und nicht den Verlust misst. Für Produkte mit einer vertraglich vereinbarten Bruttobeitragsgarantie gilt $\mu^{c} \geq 0$ und $\mu^{r} \geq 0$.

Für die Untersuchung der Abbildungen im Klassifizierungsalgorithmus führen wir einen mathematischen Rahmen ein. Da für die CRK Betrachtung die Endvermögen von Altersvorsorge relevant sind, definieren wir ein Altersvorsorgeprodukt mit simulierter Ansparphase $T$ abstrakt als Abbildung $V: \Omega \rightarrow R_{0}^{+}$, wobei $\Omega$ eine feste Menge an Kapitalmarktszenarien ist und $V(\omega)$ das Endvermögen zur Realisierung 


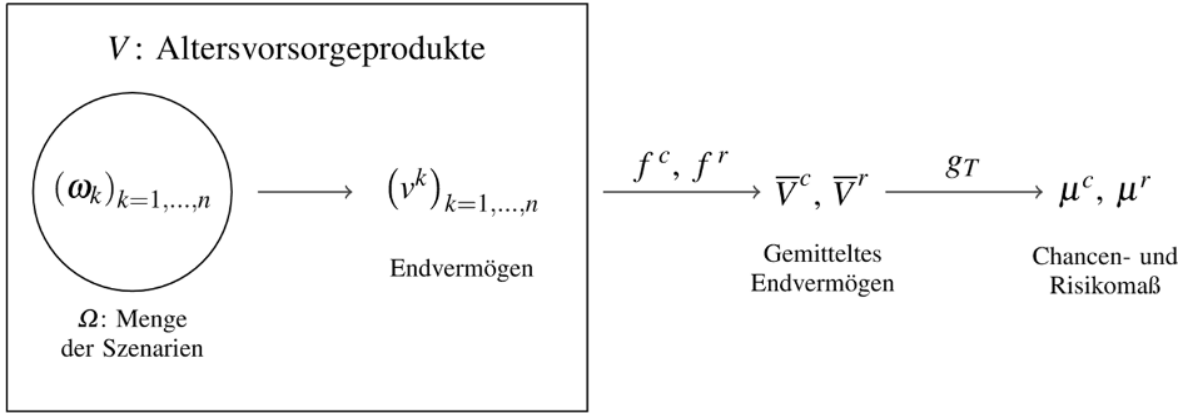

Abb. 2 Veranschaulichung der mathematischen Abbildungen

$\omega \in \Omega$. Es gilt $v^{k}=V\left(\omega_{k}\right)$. Die Addition nichtnegativer Vielfacher zweier Altersvorsorgeprodukte bildet wieder ein Altersvorsorgeprodukt, da sich die Endvermögen für jede Realisierung entsprechend addieren lassen. Die Menge der Altersvorsorgeprodukte mit einer simulierten Ansparphase von $T$ bezeichnen wir mit $\mathscr{V}_{T} . \bar{V}^{c}$ ist das Ergebnis der Abbildung $f^{c}: V_{T} \rightarrow R_{0}^{+}$mit $f^{c}(V)=\mathbb{E}(V)$ und $\bar{V}^{r}$ das Ergebnis der Abbildung $f^{r}: V_{T} \rightarrow R_{0}^{+}$mit $f^{r}(V)=\mathbb{E}\left(V \mid V \leq Q_{0,2}\right)$. $\mu^{c}$ bzw. $\mu^{r}$ werden durch $g_{T} \circ f^{c}: V_{T} \rightarrow[-12 ; \infty)$ bzw. $g_{T} \circ f^{r}: V_{T} \rightarrow[-12 ; \infty)$ mit $g_{T}: R_{0}^{+} \rightarrow[-12 ; \infty)$ bestimmt. Die verschiedenen Funktionen sind in Abb. 2 dargestellt. Mathematisch gesehen handelt es sich bei den Abbildungen $f^{c}, f^{r}, g_{T} \circ f^{c}$ und $g_{T} \circ f^{r}$ um Risikomaße.

Für die Eigenschaften der Abbildungen führen wir den Begriff der Konkavität ein, auf die die Abbildungen untersucht werden.

Definition 1 (Konkavität) Eine Abbildung $\rho: V_{T} \rightarrow \mathbb{R}$ ist konkav, wenn für $X, Y \in V_{T}$ und $0 \leq \lambda \leq 1$ gilt:

$$
\rho(\lambda X+(1-\lambda) Y) \geq \lambda \rho(X)+(1-\lambda) \rho(Y) .
$$

Bemerkung 1 Linearität von Altersvorsorgeprodukten: Würden bei einem Altersvorsorgeprodukt keinerlei Kosten anfallen, sondern die vollen Bruttobeiträge direkt in entsprechende Anlagen investiert werden, so würde ein Vielfaches $\lambda$ der Beiträge auch zu einem entsprechenden Vielfachen des Endvermögens führen. Da allerdings neben zum Vermögen proportionalen Kostenkomponenten auch Fixkosten (z. B. Depotgebühren) oder auch Kosten, die von der Anlagestrategie innerhalb des Produkts abhängen können, existieren, ist die Annahme einer solchen Linearität nur approximativ erfüllt. Je höher die Beiträge, desto geringer wird der Einfluss von Fixkosten, so dass man wieder näher an der Linearität ist. Im Fall der Konvexkombination zweier Altersvorsorgeprodukte eines Anbieters könnte dieser die Attraktivität eines solchen Portfolios für den Kunden erhöhen, indem die Fixkosten im Portfolio des Kunden nicht doppelt berechnet werden. Dann wären die Fixkosten des Portfolios gleich denen der jeweiligen Einzelprodukte. Man wäre damit in einer Konvexkombination sehr nahe an der Linearität der Kosten. Wir wollen im Folgenden immer vereinfachend annehmen, dass Linearität des Endvermögens in den Prämienzahlungen vorliegt. 
Betrachten wir unter der Annahme der Linearität $X$ und $Y$ als Endvermögen zweier Altersvorsorgeprodukte mit Einmalbeitrag oder konstanter laufender Beitragszahlung. Teilt der Kunde sein (monatlich) für die Altersvorsorge zur Verfügung stehendes Vermögen auf, dass er den Anteil $0 \leq \lambda \leq 1$ in das erste Produkt und den Rest in das zweite Produkt investiert, im festen Verhältnis $\lambda$ zwischen den beiden Produkten auf, er also diversifiziert, so erhält er ein Endvermögen von $\lambda X+(1-\lambda) Y$. Dabei sollte sich das Risiko durch die Aufteilung des Vermögens in die verschiedenen Produkte nicht erhöhen. Dies wird durch die Konkavität der Abbildungen auf die Risikomaße gewährleistet und stellt den gewünschten Diversifikationseffekt dar.

Lemma 1 Sei $f^{c}: V_{T} \rightarrow R_{0}^{+}$gemäß Gl. 1 definiert und $f^{r}: V_{T} \rightarrow R_{0}^{+}$gemäß Gl. 2. Dann ist $f^{c}$ linear und $f^{r}$ konkav.

Beweis Der Erwartungswert und somit $f^{c}(V)$ sind linear.

Zum Nachweis der Konkavität von $f^{\prime}(V)$ beachte man, dass der Mittelwert über die 2000 niedrigsten Endvermögen der gewichteten Summe der Endvermögen $X, Y \in$ $V_{T}$ zweier Produkte größer oder gleich dem Mittelwert der gewichteten Summe der jeweils 2000 niedrigsten Endvermögen der einzelnen Produkte ist. Für $0 \leq \lambda \leq 1$ mit $Z=\lambda X+(1-\lambda) Y$ gilt somit

$$
\sum_{\omega \in\left\{Z(\omega) \leq Q_{0,2}(Z)\right\}} Z(\omega) \geq \sum_{\omega \in\left\{X(\omega) \leq Q_{0,2}(X)\right\}} \lambda X(\omega)+\sum_{\omega \in\left\{Y(\omega) \leq Q_{0,2}(Y)\right\}}(1-\lambda) Y(\omega) .
$$

Hieraus folgt aufgrund der Gleichgewichtung bei der Mittelwertbildung für alle $\omega \in \Omega$ direkt die behauptete Beziehung

$$
\begin{gathered}
\mathbb{E}\left(a X+(1-\lambda) Y \mid a X+(1-\lambda) Y \leq Q_{0.2}(a X+(1-\lambda) Y)\right) \geq \\
\lambda \mathbb{E}\left(X \mid X \leq Q_{0.2}(X)\right)+(1-\lambda) \mathbb{E}\left(Y \mid Y \leq Q_{0.2}(Y)\right) .
\end{gathered}
$$

Folglich ist $f^{r}(V)$ konkav.

Bemerkung 2 Die Konkavität von $f^{\prime}(X)$ ergibt sich auch direkt aus der Konvexität des sogenannten Tail Value at Risk finanzieller Risikomaße, der sich durch Multiplikation von $f^{\prime}(X)$ mit -1 ergibt (siehe hierzu z. B. Acerbi und Tasche 2002). $\mu^{c}$ und $\mu^{r}$ werden durch die Abbildungen $g_{T} \circ f^{c}$ und $g_{T} \circ f^{r}$ bestimmt. $g_{T}: R_{0}^{+} \rightarrow[-12 ; \infty)$ ist die Umkehrfunktion zur - je nach Beitragszahlungsweise relevanten - Funktion auf der rechten Seite von Gl. 3. Diese Gleichung kann für laufende Beitragszahlung im Gegensatz zum Einmalbeitragsfall nicht explizit nach $\mu$ aufgelöst werden. Daher analysieren wir die Umkehrfunktion $g_{T}^{-1}:[-12 ; \infty) \rightarrow R_{0}^{+}$, die durch die Gl. 3 definiert ist. Da die Abbildung $\bar{V}$ für $\mu \geq-12$ streng monoton wachsend ist, ist auch ihre Inverse streng monton wachsend. Wie sich leicht zeigen lässt, sind die erste und zweite Ableitung von $g_{T}^{-1}(\mu)$ nicht-negativ für $\mu \geq-12$ sowohl für laufende als auch einmalige Beitragszahlungsweise. Des Weiteren nehmen beide Ableitungen den Wert null nur im Punkt $\mu=-12$ an. Folglich ist $g_{T}^{-1}(\mu)$ streng konvex für $\mu \geq-12$ in beiden Fällen, was dazu führt, dass $g_{T}(\bar{V})$ streng konkav für $\bar{V} \geq 0$ ist. Darüber hinaus ist $g_{T}^{-1}(\mu)$ stetig. Aufgrund der strengen Monotonie 
Abb. 3 Veranschaulichung von $g_{T}(\bar{V})$ für verschiedene Beitragszahlungsweisen und Laufzeiten

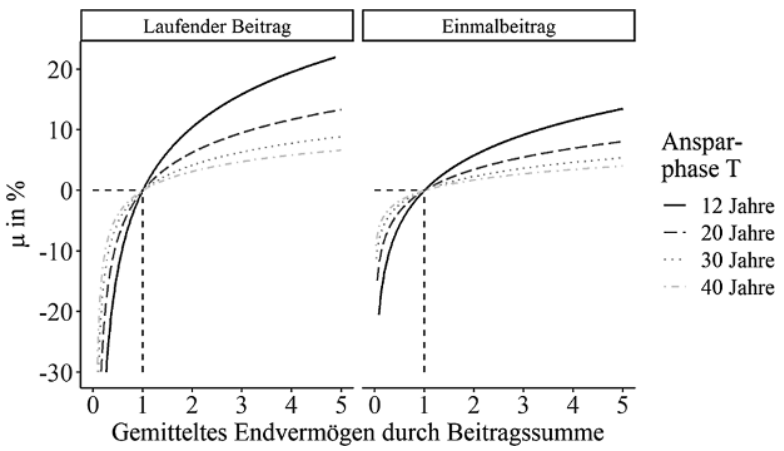

von $g_{T}^{-1}(\mu)$ für $\mu \geq-12$ ist $g_{T}(\bar{V})$ ebenfalls stetig für $\bar{V} \geq 0$. Abb. 3 veranschaulicht die Funktion $g_{T}(\bar{V})$ für mit der Beitragsumme normiertem $\bar{V} \geq 0$ und für die verschiedenen Ansparphasen $T$.

Lemma 2 Sei $f^{c}: V_{T} \rightarrow R_{0}^{+}$gemäß Gl. $1, f^{r}: V_{T} \rightarrow R_{0}^{+}$gemäß Gl. 2 und $g_{T}^{-1}: R_{0}^{+} \rightarrow[-12 ; \infty)$ gemäß Gl. 3 definiert. Dann sind $g_{T} \circ f^{c}$ und $g_{T} \circ f^{r}$ konkav.

Beweis Es seien $X, Y \in V_{T}$ und $0 \leq \lambda \leq 1$. Nach Lemma 1 ist $f^{c}$ linear während $f^{\prime}$ konkav ist. Für beide Abbildungen gilt

$$
f(\lambda X+(1-\lambda) Y) \geq \lambda f(X)+(1-\lambda) f(Y)
$$

mit $f=f^{c}, f^{r}$, wobei für $f=f^{c}$ sogar Gleichheit gilt. Aufgrund der Monotonie und Konkavität von $g_{T}$ folgt

$$
\begin{gathered}
\left(g_{T} \circ f\right)(\lambda X+(1-\lambda Y))=g_{T}(f(\lambda X+(1-\lambda) Y)) \\
\geq g_{T}(\lambda f(X)+(1-\lambda) f(Y)) \geq \lambda g_{T}(f(X))+(1-\lambda) g_{T}(f(Y)) \\
=\lambda\left(g_{T} \circ f\right)(X)+(1-\lambda)\left(g_{T} \circ f\right)(Y) .
\end{gathered}
$$

Folglich ist die Abbildung $g_{T} \circ f$ konkav.

\section{Chancen-Risiko Klasse eines Portfolios und der Einfluss auf die Kundenberatung}

Für die Kundenberatung zu Altersvorsorgeprodukten hat das Europäische Institut für Qualitätsmanagement finanzmathematischer Produkte und Verfahren (EI-QFM) in Kaiserslautern ein Verfahren entwickelt, um dem Kunden eine CRK zuzuweisen (für Details siehe Korn und Andelfinger 2016). Diskussionen in der Fachgruppe Beratungsprozesse des EI-QFM zeigten, dass es in der deutschen Versicherungswirtschaft scheinbar der Ansatz ist, dem Kunden nur Altersvorsorgeprodukte mit gleicher oder niedrigerer CRK als der CRK des Kunden anzubieten. Dieser Ansatz berücksichtigt jedoch keine Altersvorsorgeprodukte, die der Kunde bereits besitzt. 
Dadurch werden mögliche Diversifikationseffekte ignoriert. Hier stellt sich aber zum einen erst einmal die Frage, ob überhaupt ein Diversifikationseffekt im Hinblick auf die Klassifizierung vorliegt und zum anderen, wie die CRK eines Portfolios in einer Beratung zu bestimmen ist. Dazu untersuchen wir zunächst die Klassifizierung nach PIA auf Diversifikationseffekte. Basierend auf diesen Ergebnissen betrachten wir die Bestimmung der CRK eines Portfolios. Hier werden sowohl ein Portfolio, das aus zwei neu zu erwerbenden Produkten besteht, als auch ein Portfolio, das aus einem seit $m$ Monaten laufenden und einem neuen Produkt besteht, untersucht. Da die obigen Fragen in der praktischen Anwendung auftauchen und für die Versicherungswirtschaft von großem Interesse sind, leiten wir aus unseren theoretischen Erkenntnissen praktische Implikationen für die Kundenberatung ab.

Wir betrachten zwei Altersvorsorgeprodukte mit Ansparphase $T$ und gleicher Beitragszahlungsweise: Produkt $V^{i} \in V_{T}, i=1,2$, besitzt eine CRK von $C R K^{i}$. Es ist durch die Paare $\left(\bar{V}^{c, i}, \bar{V}^{r, i}\right)$ und $\left(\mu^{c, i}, \mu^{r, i}\right)$ beschrieben, die aus der Klassifizierung resultieren. Wir nehmen an, dass $C R K^{1} \leq C R K^{2}$. Die Risikopräferenz des Kunden ist mit $C R K^{K d}$ bezeichnet. Weiterhin nehmen wir an, dass die CRK-Grenzen über $b_{T}^{j}, j=1, \ldots, 4$, bekannt sind.

Die Beziehung $C R K^{1} \leq C R K^{2}$ ist gegeben, wenn die Chancen- und die Risikomaße beider Produkte in einer der folgenden Beziehungen stehen:

1. $\mu^{c, 1}<\mu^{c, 2}$ und $\mu^{r, 1}>\mu^{r, 2}$,

2. $\mu^{c, 1} \leq \mu^{c, 2}$ und $\mu^{r, 1} \leq \mu^{r, 2}$,

3. $\mu^{c, 1} \geq \mu^{c, 2}$ und $\mu^{r, 1} \geq \mu^{r, 2}$

Wir beschränken unsere Betrachtungen auf den ersten Fall, da im zweiten und dritten Fall ein Produkt das andere dominiert. Hier sollte der Gesamtbeitrag in das dominierende Produkt investiert werden.

Im Folgenden wird ein Portfolio zweier Altersvorsorgeprodukte betrachtet. Dieses wird durch die Aufteilung der Beiträge im Verhältnis $(\alpha, 1-\alpha), 0 \leq \alpha \leq 1$, in die beiden Produkte bestimmt. Wir gehen von einem Gesamtbeitrag in Höhe des Beitrages des Musterkunden aus. Für $\alpha=1$ bzw. $\alpha=0$ ergeben sich die gemittelten Endvermögen $\bar{V}^{c, i}$ und $\bar{V}^{r, i}$ sowie das jeweilige Chancenmaß $\mu^{c, i}$ und Risikomaß $\mu^{r, i}, i=1,2$. Aufgrund der von uns angenommen Linearität des Endvermögens in der Beitragshöhe entspricht die Aufteilung des Gesamtbeitrages in zwei Altersvorsorgeprodukte der gleichen Aufteilung der Endvermögen (vgl. auch Bemerkung 1 aus dem letzten Abschnitt). Somit ist das Portfolio durch $\alpha V^{1}+(1-$ $\alpha) V^{2}$ definiert.

\subsection{Diversifikationseffekt}

Ausgehend von den Eigenschaften der Chancen- und der Risikoparameter des letzten Abschnitts untersuchen wir die Klassifizierung gemäß PIA auf einen Diversifikationseffekt. Die gemittelten Endvermögen, das Chancen- und das Risikomaß des Portfolios werden anhand der Chancen- und der Risikoparameter der Altersvorsorgeprodukte abgeschätzt. Die Ergebnisse sind im folgenden Lemma zusammengefasst. 
Lemma 3 Seien $V^{i} \in V_{T}$ für $i=1,2$ mit $\bar{V}^{c, i}, \bar{V}^{r, i}$ nach Gl. 1 bzw. Gl. 2 und $\mu^{c, i}, \mu^{r, i}$ gemäß Gl. 3 abhängig von der Beitragszahlungsweise definiert. Sei $g_{T}^{-1}:[-12, \infty) \rightarrow R_{0}^{+}$gemäß Gl. 3 und das Portfolio mit dem Anteil $0 \leq \alpha \leq 1$ über die szenarioabhängigen Endvermögen definiert als $\alpha V^{1}+(1-\alpha) V^{2}$. Weiter sind die interpolierten Endvermögen bzw. Maße definiert über $\bar{V}^{j \text {,int }(\alpha)}:=\alpha \bar{V}^{j, 1}+$ $(1-\alpha) \bar{V}^{j, 2}$ bzw. $\mu^{j, \text { int }(\alpha)}:=\alpha \mu^{j, 1}+(1-\alpha) \mu^{j, 2}, j=c, r$.

a) Für das Portfolio gilt

$$
\begin{gathered}
\bar{V}^{c, \operatorname{ptf}(\alpha)}=\bar{V}^{c, \operatorname{int}(\alpha)}, \bar{V}^{r, \operatorname{ptf}(\alpha)} \geq \bar{V}^{r, \operatorname{int}(\alpha)}, \\
\mu^{c, \operatorname{ptf}(\alpha)} \geq \mu^{c, \operatorname{int}(\alpha)}, \mu^{r, \operatorname{ptf}(\alpha)} \geq \mu^{r, \operatorname{int}(\alpha)} .
\end{gathered}
$$

b) Weiterhin haben wir

$$
\bar{V}^{c, \operatorname{ptf}(\alpha)} \geq \bar{V}^{r, \operatorname{ptf}(\alpha)}, \mu^{c, \operatorname{ptf}(\alpha)} \geq \mu^{r, \operatorname{ptf}(\alpha)} .
$$

c) Für das Chancenmaß des Portfolios gilt

$\mu^{c, \operatorname{ptf}(\alpha)} \leq \max \left(\mu^{c, 1} ; \mu^{c, 2}\right)$.

d) Wir erhalten die folgenden Beziehungen zwischen den gemittelten Endvermögen, dem Chancen- und dem Risikomaß:

$$
\mu^{c, \operatorname{ptf}(\alpha)}=g_{T}\left(\bar{V}^{c, \operatorname{int}(\alpha)}\right) \geq \mu^{c, \operatorname{int}(\alpha)}, \mu^{r, \operatorname{ptf}(\alpha)} \geq g_{T}\left(\bar{V}^{r, \operatorname{int}(\alpha)}\right) \geq \mu^{r, \operatorname{int}(\alpha)}
$$

Beweis a) folgt aus der Linearität von $\bar{V}^{c}$ und der Konkavität von $\bar{V}^{r}, \mu^{c}$ sowie $\mu^{r}$.b) folgt aus der Definition von $\bar{V}^{r}$ und $\mu^{r}$. Für $c$ ) betrachten wir die Berechnung von $\mu^{c, p t f(\alpha)}$ durch

$$
\begin{aligned}
\mu^{c, \mathrm{ptf}(\alpha)} & =g_{T}(\underbrace{g_{T}^{-1}\left(\mu^{c, \mathrm{ptf}(\alpha)}\right)}_{\bar{V}^{c, \mathrm{ptf}(\alpha)}})=g_{T}(\alpha \underbrace{g_{T}^{-1}\left(\mu^{c, 1}\right)}_{\bar{V}^{c, 1}}+(1-\alpha) \underbrace{g_{T}^{-1}\left(\mu^{c, 2}\right)}_{\bar{V}^{c, 2}}) \\
& =g_{T}\left(\bar{V}^{c, \operatorname{int}(\alpha)}\right) .
\end{aligned}
$$

Ableiten von $\mu^{c, p t f(\alpha)}$ nach $\alpha$ ergibt

$$
\frac{\partial}{\partial \alpha} \mu^{c, \operatorname{ptf}(\alpha)}=\frac{\partial g_{T}\left(\bar{V}^{c, \operatorname{int}(\alpha)}\right)}{\partial \bar{V}^{c, \operatorname{int}(\alpha)}}\left(g_{T}^{-1}\left(\mu^{c, 1}\right)-g_{T}^{-1}\left(\mu^{c, 2}\right)\right) .
$$


Da $g_{T}$ und $g_{T}^{-1}$ streng monoton steigend sind, steigt $\mu^{c, p t f(\alpha)}$ in $\alpha$, wenn $\mu^{c, 1}>\mu^{c, 2}$ bzw. $\bar{V}^{c, 1}>\bar{V}^{c, 2}$, und fällt, wenn $\mu^{c, 1}<\mu^{c, 2}$ bzw. $\bar{V}^{c, 1}<\bar{V}^{c, 2}$. Folglich gilt

$$
\mu^{c, \operatorname{ptf}(\alpha)} \leq \max \left(\mu^{c, 1} ; \mu^{c, 2}\right)
$$

d) folgt aufgrund der Linearität von $\bar{V}^{c}$ und der Konkavität von $\bar{V}^{r}$ und $g_{T}$.

Das Chancenmaß des Portfolios ist - im Gegensatz zum Mittelwert der Endvermögen - nicht linear in den einzelnen Chancenmaßen der Produkte. Die Linearität des Mittelwerts der Endvermögen können wir jedoch verwenden, um $\mu^{c, p t f(\alpha)}$ zu bestimmen, vgl. Lemma $3 d$ ).

Die Linearkombination von $\bar{V}^{r}$ und $\mu^{r}$ beider Produkte stellt eine Obergrenze des Risikos des Portfolios dar. Darüber hinaus ist $\mu^{c, p t f(\alpha)}$ nach unten durch die Interpolation von $\bar{V}^{c}$ der Produkte begrenzt. Das Risiko wird reduziert, während die Ertragschance je nach betrachtetem Parameter konstant ist oder steigt. Dies ist ein klassischer Diversifikationseffekt, der aufgrund der Definition von Chance und Risiko bei der Klassifizierung von Altersvorsorgeprodukten durch die PIA besteht.

(a) $\bar{V}^{c}$ und $\bar{V}^{r}$

(b) $\mu^{c}$ und $\mu^{r}$

In Abb. 4 ist Lemma 3. veranschaulicht. Abb. 4a basiert auf den gemittelten Endvermögen $\bar{V}^{c}$ und $\bar{V}^{r}$, während Abb. 4b auf Chancenmaß $\mu^{c}$ und Risikomaß $\mu^{r}$ basiert. Die schwarze, gestrichelte Linie stellt die Interpolation der entsprechenden Chancen- und Risikoparameter für alle $\alpha \in[0 ; 1]$ dar. Die graue, dick gestrichelte Linie ist eine mögliche Realisierung von $\bar{V}^{c, \operatorname{ptf}(\alpha)}$ und $\bar{V}^{r, \operatorname{ptf}(\alpha)}$ bzw. $\mu^{c, p t f(\alpha)}$ und $\mu^{r, p t f(\alpha)}$ für $\alpha \in[0 ; 1]$.

Wir betrachten eine Linearkombination beider Produkte, die durch ein $\alpha$ bestimmt ist. In Abb. 4a sind die interpolierten $\left(\bar{V}^{c, \text { int }(\alpha)}, \bar{V}^{r, \text { int }(\alpha)}\right)$-Kombinationen vertikal nach oben $\mathrm{zu}$ verschieben, um die tatsächlichen $\left(\bar{V}^{c, \operatorname{ptf}(\alpha)}, \bar{V}^{r, \operatorname{ptf}(\alpha)}\right) \mathrm{zu}$ erhalten. Die Abschätzung nach oben von $\bar{V}^{r, \operatorname{ptf}(\alpha)}$ durch $\bar{V}^{c, \operatorname{int}(\alpha)}$ auf der y-Achse resultiert aus b) in Kombination mit a) aus Lemma 3. Abb. 4b veranschaulicht die Beziehung zwischen den interpolierten Chancen- und Risikomaßen und den tatsächlichen des Portfolios. Alle möglichen tatsächlichen Chancen- und Risikomaße des Portfolios mit einem bestimmten Anteil $\alpha$ liegen auf der grauen, dick gestrichelten Linie zwischen den zwei Pfeilen. $\mu^{r, p t f(\alpha)}$ wird nach oben durch $\mu^{c, 2}$ auf der y-Achse abgeschätzt. Dies ergibt sich aus a) und b) aus Lemma 3.

\subsection{Portfolio aus zwei neu zu erwerbenden Altersvorsorgeprodukten}

In diesem Abschnitt wird die CRK eines Portfolios bestehend aus zwei Altersvorsorgeprodukten auf Grundlage der Ergebnisse des letzten Abschnitts bestimmt. Wenn die Verteilungen der Endvermögen der einzelnen Produkte bekannt sind, erhält man die CRK des Portfolios über das gewichtete Summieren der Endvermögen jeder Simulation (immer unter der Annahme der (zumindest approximativen) Linearität der Kosten). Auf Basis dieser werden das Chancen- und das Risikomaß gemäß Abschn. 2.1 berechnet, und man erhält die CRK des Portfolios. Die Endver- 
Abb. 4 Abschätzung der Chancen- und Risikoparameter des Portfolios
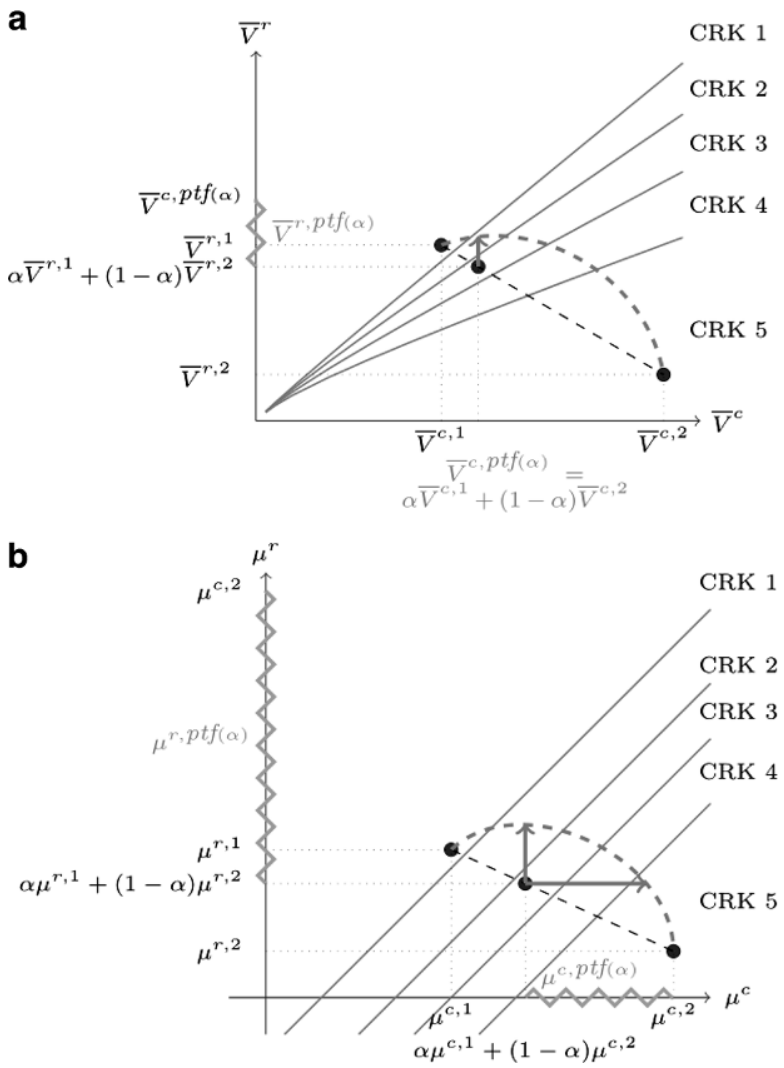

mögensverteilungen liegen den Unternehmen vor, dürfen aber laut AltvPIBV nicht veröffentlicht werden. Damit ist eine Auswertung im Beratungsprozess nicht möglich und den Beratern liegen lediglich die CRK der Produkte vor, die auf den PIB abgedruckt sind. Diese Information ist für die CRK-Ermittlung des Portfolios nicht ausreichend, wie wir im Folgenden sehen werden.

Lemma 3 ermöglicht es uns, die CRK des Portfolios abzuschätzen, was uns zu folgendem Theorem führt.

Theorem 1 Es seien $V^{i} \in V_{T}$ mit $\bar{V}^{c, i}, \bar{V}^{r, i}, i=1,2$, gemäß Gl. 1 und 2 und $g_{T}^{-1}:[-12, \infty) \rightarrow R_{0}^{+}$gemäß Gl. 3 definiert. Sei $0 \leq \alpha \leq 1$. Die CRK des Portfolios auf Basis der gewichteten Endvermögenverteilungen ist nicht größer als die CRK, die sich aus der Interpolation der gemittelten Endvermögen $\bar{V}^{c, i}$ und $\bar{V}^{r, i}$ der einzelnen Produkte mit Anteil $\alpha$ ergibt.

Beweis Die CRK-Grenze zwischen CRK $j$ und $j+1, j=1,2,3,4$ ist als Gerade mit Steigung 1 definiert. Diese transformieren wir mit $g_{T}^{-1}$ und bezeichnen die resultierende Funktion mit $l_{j, T}: R_{0}^{+} \rightarrow R_{0}^{+}$. Da $g_{T}^{-1}$ monoton wachsend ist, ist auch $l_{j, T}$ monoton wachsend. 
Mit Lemma 3 gilt für die gemittelten Endvermögen, dass $\bar{V}^{c, \operatorname{ptf}(\alpha)}$ gleich der Interpolation von $\bar{V}^{c}, 1$ und $\bar{V}{ }^{c}, 2$ mit Anteil $\alpha$ ist, während $\bar{V} r$,ptf $(\alpha)$ größer als die Interpolation von $\bar{V}^{r, 1}$ und $\bar{V}^{r, 2}$ ist. Im $\mathbb{R}^{2}$ liegt der Punkt $\left(\bar{V}^{c, \operatorname{ptf}(\alpha)}, \bar{V}^{r, \operatorname{ptf}(\alpha)}\right)$ vertikal über $\left(\bar{V}^{c, \text { int }(\alpha)}, \bar{V}^{r, \text { int }(\alpha)}\right)$. Wir unterscheiden zwei Fälle:

$$
\begin{aligned}
& \left(\bar{V}^{c, \operatorname{int}(\alpha)}, \bar{V}^{r, \operatorname{int}(\alpha)}\right) \text { liegt im Bereich oberhalb von } l_{4, T} \\
& \left(\bar{V}^{c, \operatorname{int}(\alpha)}, \bar{V}^{r, \operatorname{int}(\alpha)}\right) \text { liegt im Bereich unterhalb von } l_{4, T}
\end{aligned}
$$

Zu Fall (i): In diesem Fall gibt es ein minimales $j$, so dass $\left(\bar{V}^{c, \operatorname{int}(\alpha)}, \bar{V}^{r, \operatorname{int}(\alpha)}\right) \mathrm{im}$ Bereich oberhalb $l_{j, T}$ liegt. Der Punkt $\left(\bar{V}^{c, \operatorname{ptf}(\alpha)}, \bar{V}^{r, \operatorname{ptf}(\alpha)}\right)$ befindet sich auch in dem Bereich über $l_{j, T}$ aufgrund seiner Lage zu $\left(\bar{V}^{c, \operatorname{int}(\alpha)}, \bar{V}^{r \text {,int }(\alpha)}\right)$. Wir führen nun eine Transformation mit $g_{T}$ durch. Da $g_{T}$ stetig ist, liegen die Transformationen beider Punkte mit $g_{T}$ im Bereich über der Transformation von $l_{j, T}$. Die Transformation der Linie $l_{j, T}$ mit $g_{T}$ ist aufgrund der Wahl von $l_{j, T}$ die CRK-Grenze zwischen CRK $j$ und $j+1$. Folglich ist die CRK des Portfolios nicht größer als die CRK, die sich aus der Interpolation von $\bar{V}^{c, i}$ und $\bar{V}^{r, i}, i=1,2$, ergibt.

Zu Fall (ii): Liegt $\left(\bar{V}^{c}\right.$, int $\left.(\alpha), \bar{V}^{r, \operatorname{int}(\alpha)}\right)$ im Bereich unterhalb $l_{4, T}$, dann liegt die Transformation von $\left(\bar{V}^{c, \text { int }(\alpha)}, \bar{V}^{r \text {,int }(\alpha)}\right)$ mit $g_{T}$ unterhalb der CRK-Grenze zwischen CRK 4 und 5 aufgrund der Definition von $l_{4, T}$ und der Stetigkeit von $g_{T}$. Damit ergibt sich aus der Interpolation der gemittelten Endvermögen eine CRK von 5. Da es sich hierbei um die größte CRK handelt, kann die CRK des Portfolios nicht größer sein als die CRK, die sich aus der Interpolation von $\bar{V}^{c, i}$ und $\bar{V}^{r, i}$, $i=1,2$, ergibt.

Bemerkung 3 Aufgrund der zusätzlichen horizontalen Verschiebung von $\left(\bar{V}^{c, \operatorname{ptf}(\alpha)}, \bar{V}^{r, \operatorname{ptf}(\alpha)}\right)$ zu $\left(\bar{V}^{c, \operatorname{int}(\alpha)}, \bar{V}^{r, \operatorname{int}(\alpha)}\right)$ kann die Aussage von Theorem 1 nicht auf die Interpolation der Chancen- und der Risikomaße der einzelnen Produkte übertragen werden. Die CRK, die sich aus der Interpolation der Chancen- und der Risikomaße der einzelnen Produkte ergeben würde, ist also nicht notwendigerweise eine obere Grenze für die tatsächliche CRK des Portfolios.

Wir bezeichnen die CRK zur Interpolation der gemittelten Endvermögen mit $\mathscr{C} \mathscr{R} \mathscr{K} . \mathscr{C} \mathscr{R} \mathscr{K}^{p t f^{\prime}(\alpha)}$ ist dann die CRK zum Portfolio $\alpha V^{1}+(1-\alpha) V^{2} \cdot \mathscr{C} \mathscr{R} \mathscr{K}^{p t f^{\prime}(\alpha)}$ entspricht $C R K^{1}$ für $\alpha=1$ und $C R K^{2}$ für $\alpha=0$.

Empfehlungen für die Kundenberatung 1 Die Bedeutung von Theorem 1 für die praktische Anwendung ist eindeutig. Für die näherungsweise Bestimmung der CRK des Portfolios im Sinne einer Abschätzung der CRK nach oben sind die gemittelten Endvermögen zu verwenden. Eine exakte Bestimmung der CRK des Portfolios wäre nur durch eine Neusimulation der zugehörigen Endvermögen möglich, eine approximative Bestimmung im Sinne der von uns getroffenen Annahme der Linearität in den Endvermögen mit Hilfe der bereits jeweils vorliegenden simulierten Endvermö- 
gen der einzelnen Altersvorsorgeprodukte. Die Interpolation der Chancen- und der Risikomaße oder sogar nur der CRK zur Bestimmung der CRK des Portfolios ist hingegen gemäß Bemerkung 3 nicht zulässig, da sie zu einer fehlerhaften Beratung führen können. Darf man dem Kundenberater auch in Zukunft nicht die gemittelten Endvermögen für die Bestimmung der einzelnen Chancen- und Risikomaße zugänglich machen, so könnte ihm der Anbieter zumindest eine einfache Software zur Abschätzung der CRK bei gegebenem Portfolioparameter $\alpha$ zur Verfügung stellen. Allerdings gilt es hier zu bedenken, dass dies ein Anbieter nur für Portfolios aus seinen eigenen Produkten liefern kann, da ihm die gemittelten Endvermögen der Produkte von Fremdanbietern nicht bekannt sind.

Abb. 4 zeigt auch die Relation zwischen der CRK resultierend aus der Interpolation der Chancen- und Risikoparameter und der CRK des Portfolios. Wenn man die gemittelten Endvermögen wie in Abb. 4a heranzieht, besitzt das Portfolio CRK 2, wenn man annimmt, dass die graue, dick gestrichelte Linie die tatsächliche Realisierung von $\bar{V}^{c, \operatorname{ptf}(\alpha)}$ und $\bar{V}^{r, \operatorname{ptf}(\alpha)}$ in Abhängigkeit von $\alpha$ ist. Niedrigere CRK sind durch einen größeren vertikalen Shift der Linearkombination nach oben möglich. Offensichtlich kann keine CRK größer als 3, die $\mathscr{C} \mathscr{R} \mathscr{K} p t f(\alpha)$ entspricht, erzeugt werden. Abb. 4b betrachtet die Interpolation des Chancen- und des Risikomaßes. Alle möglichen Chancen- und Risikomaße des Portfolios liegen auf der grauen, dick gestrichelten Linie zwischen den beiden Pfeilen. Offensichtlich kann das Portfolio sowohl eine niedrigere als auch höhere CRK haben als die CRK der interpolierten Chancen- und Risikomaße, die gleich CRK 3 ist.

Als nächstes untersuchen wir die Beziehung zwischen dem Anteil $\alpha$ und der CRK des Portfolios.

Theorem 2 Es seien $V^{i} \in V_{T}$ mit $\mu^{c, i}, \mu^{r, i}, i=1,2$, berechnet nach $f \circ g_{T}$, wobei $f$ gemäß Gl. 1 bzw. 2 und $g_{T}^{-1}$ gemäß Gl. 3 definiert sind. Weiter seien $\mu^{c, 1}<\mu^{c, 2}$, $\mu^{r, 1}>\mu^{r, 2}$ so gewählt, dass $C R K^{1} \leq C R K^{2}$. Sei $C R K^{K d}$ eine gegebene Risikopräferenz mit $C R K^{1} \leq C R K^{K d} \leq \bar{C} R K^{2}$ und $b_{T}^{C R K^{K d}} \operatorname{der} \mu^{c}$-Achsenabschnitt der CRK-Grenze zwischen $C R K^{K d}$ und $C R K^{K d}+1$ für die Ansparphase T. Die Prämienaufteilung $\alpha^{*}$ sei so gewählt, dass $\alpha^{*}$ die folgende Gleichung löst

$$
\begin{gathered}
g_{T}\left(\alpha^{*} g_{T}^{-1}\left(\mu^{c, 1}\right)+\left(1-\alpha^{*}\right) g_{T}^{-1}\left(\mu^{c, 2}\right)\right) \\
-g_{T}\left(\alpha^{*} g_{T}^{-1}\left(\mu^{r, 1}\right)+\left(1-\alpha^{*}\right) g_{T}^{-1}\left(\mu^{r, 2}\right)\right) \\
= \begin{cases}\mu^{c, 2}-\mu^{r, 2} & C R K^{K d}=C R K^{2} \\
b_{T}^{C R K} K^{K d} & \text { sonst. }\end{cases}
\end{gathered}
$$

Dann ist für $\alpha>\alpha^{*}$ die CRK des Portfolios nicht größer als $C R K^{K d}$.

Beweis Wir verwenden die Abschätzung $d$ ) in Lemma 3 und erhalten

$$
\mu^{c, \operatorname{ptf}(\alpha)}-\mu^{r, \operatorname{ptf}(\alpha)} \leq g_{T}\left(\bar{V}^{c, \operatorname{int}(\alpha)}\right)-g_{T}\left(\bar{V}^{r, \operatorname{int}(\alpha)}\right) .
$$


Wir bezeichnen die rechte Seite der Ungleichung mit $u(\alpha)$. Die Ableitung von $u(\alpha)$ nach $\alpha$ ist dann

$$
\begin{aligned}
& \frac{\partial}{\partial \alpha} u(\alpha)=\frac{\partial}{\partial \bar{V}^{c, \operatorname{int}(\alpha)}} g_{T}\left(\bar{V}^{c, \operatorname{int}(\alpha)}\right)\left(g_{T}^{-1}\left(\mu^{c, 1}\right)-g_{T}^{-1}\left(\mu^{c, 2}\right)\right) \\
& -\frac{\partial}{\partial \bar{V}^{r, \operatorname{int}(\alpha)}} g_{T}\left(\bar{V}^{r, \operatorname{int}(\alpha)}\right)\left(g_{T}^{-1}\left(\mu^{r, 1}\right)-g_{T}^{-1}\left(\mu^{r, 2}\right)\right) .
\end{aligned}
$$

Für $\mu^{c, 1}<\mu^{c, 2}$ und $\mu^{r, 1}>\mu^{r, 2}$ gilt $\frac{\partial}{\partial \alpha} u(\alpha)<0$ aufgrund der Monotonie von $g_{T}$. Folglich ist in diesem Fall $u(\alpha)$ strikt monoton fallend. Weiter gelten

$$
\begin{aligned}
& u(0)=\mu^{c, 2}-\mu^{r, 2}, \\
& u(1)=\mu^{c, 1}-\mu^{r, 1}<b_{T}^{C R K^{1}},
\end{aligned}
$$

wobei $b_{T}^{j}$ der $\mu^{c}$-Achsenabschnitt der CRK-Grenze zwischen CRK $j$ und CRK $j+1$ für die Anspardauer $T$ ist. Für $C R K^{1}<C R K^{2}$ gilt $u(0)>u(1)$, da $\mu^{c, 1}<\mu^{c, 2}$ und $\mu^{r, 1}>\mu^{r, 2}$. Für $C R K^{K d}=C R K^{2}$ löst $\alpha^{*}=0$ die Gl. 4. Aufgrund der Monotonie von $u(\alpha)$ existiert kein weiteres $\alpha$, das die Gl. 4 löst. Für $C R K^{1} \leq C R K^{K d}<$ $C R K^{2}$ gilt

$$
u(0) \geq b_{T}^{C R K^{2}-1} \geq b_{T}^{C R K^{1}}>u(1) .
$$

Kombiniert mit der strengen Monotonie von $u(\alpha)$ und der Wahl von $C R K^{K d}$ führt dies zur Existenz genau eines $\alpha^{*}$, das die Gl. 4 löst. Da $u(\alpha)$ streng monoton fällt erhalten wir die Abschätzung

$$
\mu^{c, \operatorname{ptf}(\alpha)}-\mu^{r, \operatorname{ptf}(\alpha)} \leq u(\alpha)<u\left(\alpha^{*}\right)= \begin{cases}\mu^{c, 2}-\mu^{r, 2} & C R K^{K d}=5 \\ b_{T}^{C R K^{K d}} & \text { sonst }\end{cases}
$$

für $\mu^{c, 1}<\mu^{c, 2}, \mu^{r, 1}>\mu^{r, 2}$ und $\alpha>\alpha^{*}$. Für diesen Fall ist die CRK des Portfolios mit Anteil $\alpha>\alpha^{*}$ nicht größer als $C R K^{K d}$.

Für $C R K^{K d}=C R K^{2}$ gilt $\alpha^{*}=0$. In diesem Fall ist die CRK des Portfolios zweier Produkte mit dem Anteil $\alpha$ nicht größer als $C R K^{2}$ für alle $0 \leq \alpha \leq 1$. Dies ist im Einklang mit der Erwartung, dass es nicht möglich ist, eine größere CRK zu generieren als die größte CRK beider Altersvorsorgeprodukte, wenn zwei Produkte kombiniert werden.

Die qualitativen Kriterien von CRK 1 und 2 werden in Theorem 1 und Theorem 2 nicht berücksichtigt. Falls ein Produkt diese nicht erfüllt, kann die CRK des Portfolios nicht niedriger als CRK 2 oder CRK 3 sein.

Empfehlungen für die Kundenberatung 2 Theorem 2 liefert eine Gleichung zur Bestimmung des Anteils des Gesamtbeitrages in das Produkt 1, so dass die CRK des Portfolios nicht größer als eine gewünschte CRK ist. Da diese Gleichung nicht nach $\alpha^{*}$ aufgelöst werden kann, muss $\alpha^{*}$ numerisch bestimmt werden. 
Die qualitativen Kriterien müssen wie folgt berücksichtigt werden:

- Der Kunde besitzt $C R K^{K d}=1$ : Nur Altersvorsorgeprodukte mit einer vertraglichen Bruttobeitragsgarantie und stetig ansteigendem Kapital sind zu kombinieren.

- Der Kunde besitzt $C R K^{K d}=2$ : Nur Produkte mit einer vertraglichen Bruttobeitragsgarantie sind zu kombinieren. Dies ist bei Riesterprodukten gewährleistet.

- Der Kunde besitzt $C R K^{K d}>2$ : Alle möglichen Altersvorsorgeprodukte können kombiniert werden.

Theorem 2 kann auch dazu verwendet werden, um zwei Altersvorsorgeprodukte mit unterschiedlichen CRK so zu kombinieren, dass ein neues Produkt mit einer dazwischen liegenden CRK entsteht.

Wir veranschaulichen Theorem 2 an einem Beispiel mit laufender Beitragszahlung und einer Ansparphase von 12 Jahren. Produkt 1 ist spezifiziert durch $\mu^{c, 1}=2,66 \%, \mu^{r, 1}=1,02 \%$ und eine CRK von 1 , während Produkt 2 durch $\mu^{c, 2}=8,13 \%, \mu^{r, 2}=-2,96 \%$ und einer CRK von 5 charakterisiert ist. Produkt 2 besitzt weder eine Bruttobeitragsgarantie noch ein stetig wachsendes Kapital. Offensichtlich sind die Anforderungen $\mu^{c, 1}<\mu^{c, 2}$ und $\mu^{r, 1}>\mu^{r, 2}$ erfüllt. Da keine vertragliche Bruttobeitragsgarantie im Gesamten vorliegt, können CRK 1 und 2 nicht vom Portfolio erreicht werden. Trotzdem berechnen wir zur Veranschaulichung auch $\alpha^{*}$ für $C R K^{K d}$ von 1 und 2. Lösen der Gl. 4 nach $\alpha^{*}$ für verschiedene $C R K^{K d}$ liefert die $\alpha^{*}$ der Tab. 1.

Für verschiedene $\alpha \in[0 ; 1]$ werden sowohl $\mu^{c, p t f(\alpha)}$ und $\mu^{r, p t f(\alpha)}$ als auch die konstanten Renditen beider interpolierten gemittelten Endvermögen als Schätzung von Chancen- und Risikomaß des Portfolios berechnet. Diese Parameter und die dazugehörige CRK sind in Abb. 5 verdeutlicht. Abb. 5a zeigt die Beziehung zwischen der tatsächlichen und der geschätzten CRK. Die untere Linie repräsentiert die konstante Rendite der interpolierten gemittelten Endvermögen, während die obere die tatsächlichen Werte des Portfolios zeigt. Jeder Punkt auf den Linien gehört zu einem $\alpha$. Die gleichen $\alpha$ sind durch einen vertikalen Pfeil verbunden, der die Richtung angibt, in welche die Renditen der interpolierten gemittelten Endvermögen zu verschieben sind, um die tatsächlichen zu erhalten. Der Übergang von $\alpha$ von 1 zu 0 stellt den Übergang von Produkt 1 zu 2 dar. Die Form zeigt in welchem Intervall $\alpha$ abhängig von $\alpha^{*}$ liegt, das wiederum von $C R K^{K d}$ abhängt. Wir bezeichnen das $\alpha^{*}$, das zu $C R K^{K d}=j, j=1, \ldots, 5$, gehört mit $\alpha_{j}^{*}$. Die runden Punkte sind alle $\alpha$ mit $\alpha>\alpha_{1}^{*}$. Die dreieckigen erfüllen $\alpha_{1}^{*} \geq \alpha>\alpha_{2}^{*}$ usw. Die Aussage von Theorem 2 ist hier deutlich zu erkennen.

Diese ist auch in Abb. 5b zu sehen. Sie zeigt die tatsächliche und geschätzte CRK abhängig von $\alpha$. Die vertikal gestrichelte Linien stellen $\alpha_{j}^{*}, j=1,, 5$, dar. Linksseitig in der Nähe von $\alpha_{j}^{*}$ ist ein Sprung in den CRK zu erkennen. Die tatsächliche CRK des Portfolios gemäß der Endvermögenverteilung ist kleiner als die CRK, die aus der Interpolation der gemittelten Endvermögen resultiert. Davor sind 
Tab. 1 Minimal benötigter Anteil $\alpha^{*}$ des ersten Produktes im Portfolio, um die vorgegebene CRK ${ }^{K d}$ für das Portfolio zu erzielen

\begin{tabular}{llllll}
\hline $\boldsymbol{C R} K^{K d}$ & 1 & 2 & 3 & 4 & 5 \\
\hline$\alpha^{*}$ in $\%$ & 94,86 & 75,18 & 46,56 & 0,27 & 0 \\
\hline
\end{tabular}

die CRK gleich. CRK 1 und 2 können aufgrund der fehlenden qualitativen Kriterien des Portfolios nicht erreicht werden. Dennoch zeigt Abb. 5 auch diese Werte.

(a) $\mu^{c}-\mu^{r}-$ Diagramm

(b) $\alpha$-CRK-Diagramm

\subsection{Portfolio aus einem laufenden und einem neuen Altersvorsorgeprodukt}

In diesem Abschnitt befassen wir uns mit dem Zukauf eines Altersvorsorgeproduktes und der Bestimmung der CRK des Portfolios, wenn dieses bereits ein Altersvorsorgeprodukt enthält. Der von uns vorgestellte Ansatz steht im Einklang mit dem Konzept der PIA. Die Festlegung der Portfolio CRK erfolgt wieder über die Aufteilung der Prämienzahlung.

Das bestehende Produkt wurde vor $m$ Monaten mit einem vertraglich vereinbarten Beitrag von $P^{l f d}$ und Ansparphase von $T^{f f d}$ abgeschlossen. Daneben haben wir ein neues Produkt mit einer vertraglichen Ansparphase von $T^{\text {neu. }}$. Die vertragliche Ansparphase beider Produkte endet gleichzeitig. Somit entspricht die restliche Ansparphase des laufenden Produktes der vertraglichen Ansparphase des neuen Produktes, $T^{\text {neu }}=T^{\text {lfd }}-\frac{m}{12}$. Beide Altersvorsorgeprodukte sollen die gleiche Beitragszahlungsweise besitzen. Die Klassifizierung des neuen Produktes basiert auf einer Ansparphase von $T^{\text {пеи }}$ und einer Prämie von $P_{T^{\text {пеш. }}}$. Wir nehmen an, dass die gemittelten Endvermögen aus der Klassifizierung des neuen Produktes, bezeichnet mit $\bar{V}^{c \text {, neu }}$ und $\bar{V}^{r \text {,neu }}$, sowie das Chancenmaß $\mu^{c, \text { neu }}$ und Risikomaß $\mu^{r \text {,neu }}$ neben der CRK bekannt sind. Der Kunde will einen Zusatzbeitrag von $I$ investieren. Je nach Beitragszahlungsweise handelt es sich dabei um den monatlichen Beitrag oder einen Einmalbeitrag. Wir nehmen an, dass der volle Betrag als Prämie für das neue Produkt verwendet wird, $P^{\text {neu }}=I$.

\subsubsection{Bestimmung der Chancen-Risiko Klasse}

Nach Theorem 1 kann die CRK eines Portfolios anhand der gemittelten Endvermögen nach oben abgeschätzt werden. Die gemittelten Endvermögen des neuen Produktes sind bekannt. Jedoch sind die aktuellen gemittelten Endvermögen des laufenden Produktes zu bestimmen. Deren Berechnung ist in Abb. 6 veranschaulicht.

Grundlage ist das aktuelle Vertragsvermögen $v_{m}^{\text {lfd }}$ des laufenden Altersvorsorgeproduktes. Dies kann der jährlichen Information über den Vertrag entnommen werden. Das aktuelle Vertragsvermögen ist auf die Beitragszahlung $P_{T_{\text {neu }}}$ des Mus- 
Abb. 5 Veranschaulichung der Beziehung zwischen $\alpha^{*}, \mathscr{C} \mathscr{R} \mathscr{K}$ und CRK des Portfolios

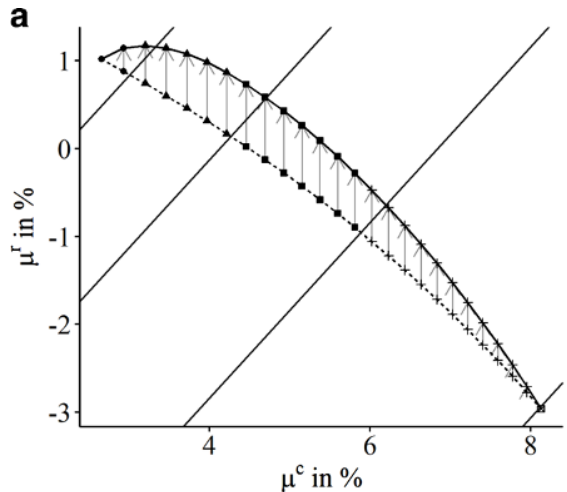

$\alpha \in$

- $\left(\alpha_{1}^{*} ; 1\right]$

- $\left(\alpha_{2}^{*} ; \alpha_{1}^{*}\right]$

- $\left(\alpha_{3}^{*} ; \alpha_{2}^{*}\right]$

$+\left(\alpha_{4}^{*} ; \alpha_{3}^{*}\right]$

๙ $\left[0 ; \alpha_{4}^{*}\right]$

Interpolation von

- Endvermögen

.... $\overline{\mathrm{V}}^{\mathrm{c}}$ und $\overline{\mathrm{V}}^{\mathrm{r}}$

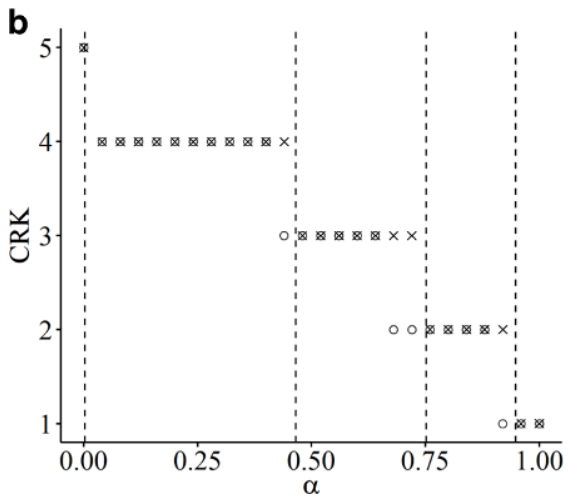

Interpolation von

- Endvermögen

$\times \overline{\mathrm{V}}^{\mathrm{c}}$ und $\overline{\mathrm{V}}^{\mathrm{r}}$

terkunden zu skalieren, um ihm eine (künstliche, aber aktualisierte) CRK zuweisen zu können,

$$
\widetilde{v}_{m}^{\mathrm{ffd}}=v_{m}^{\mathrm{lfd}} \cdot \frac{P_{T^{\mathrm{neu}}}}{P^{\mathrm{lfd}}},
$$

wobei $\widetilde{v}_{m}^{\mathrm{fd}}$ das auf den Beitrag des Musterkunden skalierte Vertragsvermögen ist und von einer linearen Abhängigkeit zwischen Beitragszahlung und Vertragsvermögen ausgegangen wird. Die Skalierung auf $P_{T^{\text {neu }}}$ ergibt sich aus der Wahl der Funktion $g_{T}$ unten (siehe Gln. 5 und 6). Darüber hinaus gewährleistet die Skalierung einen konsistenten Ansatz zu der Klassifizierung gemäß PIA. Wenn sich das Vertragsvermögen aus verschiedenen Vermögensanlagen zusammensetzt (wie z.B. bei einen Drei-Topf-Hybridprodukt), müssen alle Vermögenskomponenten wie oben beschrieben skaliert werden.

Bemerkung 4 Die CRK-Bestimmung des bereits laufenden Produkts kann nicht mehr auf der Basis der ursprünglich durch die PIA vorgenommenen Simulationen erfolgen, da ja Erträge und Kosten des Produkts während der bisherigen Laufzeit mittlerweile bekannt sind und somit nur noch eine Unsicherheit der Vermögensentwicklung über die restliche Laufzeit besteht. Außerdem haben sich evtl. auch die für die aktuelle Simulation neuer Produkte oder auch die Neu-Klassifizierung 


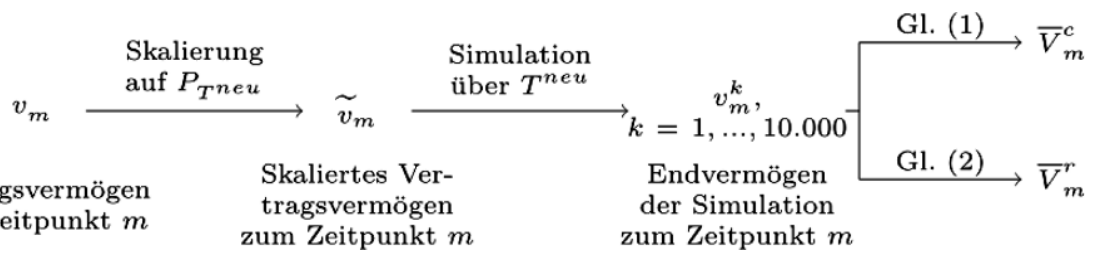

Vertragsvermögen

zum Zeitpunkt $m$

Abb. 6 Veranschaulichung der Berechnug der aktuellen gemittelten Endvermögen zum Zeitpunkt $m$ des laufenden Rentenprodukts

der aktuellen Variante des Produkts relevanten Kapitalmarktparameter der Simulation geändert, was auch für die vereinbarten Vertragsbedingungen des Produkts gegenüber seiner aktuell vertriebenen Variante gelten kann. Des Weiteren ist die Zillmerphase des Produkts bereits (teilweise) abgeschlossen und es liegt somit eine andere Kostenstruktur als bei einem neu abgeschlossenen Vertrag mit gleichem Ende der Ansparphase vor. Die PIA führt keine Simulationen für laufende Produkte durch. Eine mögliche Lösung, die evtl. der Zustimmung des Gesetzgebers bedarf, zumindest aber als allgemeine Marktpraxis anerkannt werden müsste, kann in der Simulation durch den Anbieter liegen, in dem er für den Rest der Laufzeit die Kapitalmarktentwicklungen mit den aktuell gültigen Marktparametern des PIA-Basismodells simuliert. Wir gehen im Folgenden davon aus, dass eine solche Vorgehensweise möglich ist.

Mit $\widetilde{v}_{m}^{\text {fd }}$ als Startkapital werden die Endvermögen über die gleiche Ansparphase $T^{\text {neu }}$ wie das neue Produkt simuliert. In der Simulation wird ein monatlicher Beitrag von $100 €$ als laufender Beitrag verwendet. Bei Einmalbeitragszahlung wird keine weitere Prämienzahlung herangezogen. Des Weiteren wird die Simulation auf Basis der aktuellen Finanzmarktparameter mit den aktuell gültigen Vertragskosten zum jeweiligen Zeitpunkt der Entnahmen, aber den Vertragsparametern bei Vertragsabschluss durchgeführt. Die Beitragssumme in der Simulation ist definiert als $100 \cdot\left(12 \cdot T^{\text {neu }}+m\right)$ für die laufende Beitragszahlung und $1.200 \cdot T^{\text {neu }}$ für die Einmalbeitragszahlung. Der Einmalbeitrag resultiert aus der Skalierung des Vertragsvermögens zum Zeitpunkt $m$. Folglich müssen Kosten, deren Basis die Bruttobeitragssumme ist, wie z.B. die Abschlusskosten einer klassischen Lebensversicherung, an die Bruttobeitragssumme der aktuellen Simulation angepasst werden. Die Abschlusskosten werden gewöhnlich gemäß Zillmerung über die ersten fünf Jahre verteilt. Diese sind in der Simulation zum Zeitpunkt $m \leq 60$ mit einer angepassten Bezugsgröße und zum Zeitpunkt der Entnahme zu berücksichtigen.

Als Ergebnis erhalten wir die simulierten Endvermögen. Aus diesen werden dann die gemittelten Endvermögen $\bar{V}_{m}^{c, \text { Ifd }}$ und $\bar{V}_{m}^{r \text {,Ifd }}$ des laufenden Produktes nach Gln. 1 und 2 berechnet.

Auf Basis der gemittelten Endvermögen beider Produkte können wir jetzt die CRK des Portfolios durch Interpolation der gemittelten Endvermögen nach oben abschätzen. Der Anteil $\alpha$ des Gesamtbeitrags, der in das laufende Produkt investiert wird, ergibt sich aus 


$$
\alpha=\frac{P^{\mathrm{lfd}}}{P^{\mathrm{lfd}}+P^{\text {neu }}},
$$

während $(1-\alpha)$ in das neue Altersvorsorgeprodukt investiert wird. Die interpolierten gemittelten Endvermögen ergeben sich dann durch

$$
\begin{aligned}
\bar{V}^{c, \text { int }(\alpha)} & =\alpha \bar{V}_{m}^{c, \text { lfd }}+(1-\alpha) \bar{V}^{c, \text { neu }}, \\
\bar{V}^{r, \text { int }(\alpha)} & =\alpha \bar{V}_{m}^{r \text {,lfd }}+(1-\alpha) \bar{V}^{r, \text { neu }} .
\end{aligned}
$$

Als nächstes werden das Chancen- und das Risikomaß der interpolierten gemittelten Endvermögen berechnet. Würden wir diese gemäß Gl. 3 mit $T^{\text {neu }}$ als $T$ und dem entsprechenden $\bar{V}^{\text {int }(\alpha)}$ berechnen, dann erhielten wir tendenziell ein erhöhtes Chancen- und Risikomaß aus dem Grund, dass die bereits gezahlten Beiträge des laufenden Produktes nicht berücksichtigt werden. Diese werden als Rendite behandelt. Dieser Effekt wird auch dadurch verstärkt, dass bereits die Zillmerphase mit ihren erhöhten Kosten (zumindest teilweise) abgelaufen ist. ${ }^{4}$ Folglich ist die Funktion zur Bestimmung des Chancen- und des Risikomaßes anzupassen.

Das Chancen- und das Risikomaß ist gemäß PIA definiert als jährliche konstante Rendite eines Sparprozesses über die Ansparphase T. Zu Vertragsbeginn ist $T$ sowohl die gesamte Ansparphase als auch die verbleibende Ansparphase. Folglich können das Chancen- und das Risikomaß sowohl als konstante Rendite über die gesamte Vertragslaufzeit als auch über die restliche Laufzeit gesehen werden. Beim ersten Ansatz berechnet sich das Chancen- und das Risikomaß als jährliche konstante Rendite über die gesamte Ansparphase über

$$
\bar{V}= \begin{cases}\alpha \cdot 100 \sum_{j=1}^{m}\left(1+\frac{\mu}{12}\right)^{12 T+j}+100 \sum_{j=1}^{12 T}\left(1+\frac{\mu}{12}\right)^{j} & \text { laufenderBeitrag } \\ 1.200 \cdot T\left(\alpha \cdot\left(1+\frac{\mu}{12}\right)^{12 T+m}+(1-\alpha) \cdot\left(1+\frac{\mu}{12}\right)^{12 T}\right) & \text { Einmalbeitrag. }\end{cases}
$$

Beim zweiten Ansatz lösen das Chancen- und das Risikomaß als jährliche konstante Rendite über die restliche Laufzeit die Gleichung

$$
\bar{V}= \begin{cases}\alpha \cdot \widetilde{v}_{m} \cdot\left(1+\frac{\mu}{12}\right)^{12 T}+100 \sum_{j=1}^{12 T}\left(1+\frac{\mu}{12}\right)^{j} & \text { laufenderBeitrag } \\ \left(\alpha \cdot \widetilde{v}_{m}+(1-\alpha) \cdot 1.200 \cdot T\right) \cdot\left(1+\frac{\mu}{12}\right)^{12 T} & \text { Einmalbeitrag. }\end{cases}
$$

Das Chancen- und das Risikomaß der interpolierten gemittelten Endvermögen erhält man dann durch Lösen der $G l .5$ bzw. 6 mit $T^{\text {neu }}$ als $T$, dem entsprechenden interpolierten gemittelten Endvermögen $\bar{V}^{\operatorname{int}(\alpha)}$ als $\bar{V}$ und dem entsprechenden $\mu$.

Wir definieren $g_{T, \alpha}(\bar{V})$ als Umkehrfunktion der rechten Seiten von Gl. $5 \mathrm{bzw}$. 6 aufgelöst nach $\mu$. Da $g_{T, \alpha}^{-1}(\mu)$ für $\mu \geq-12$ aus streng monoton wachsenden und konvexen Summanden besteht (vgl. Abschn. 2.3), ist $g_{T, \alpha}(\bar{V})$ wiederum streng

\footnotetext{
4 Wir verwenden hier bewusst das Adjektiv tendenziell, da wir neue Simulationen mit geänderten Kapitalmarktparametern verwenden und so trotz niedrigerer Kosten auch die Möglichkeit niedrigerer Erträge besteht.
} 
monoton wachsend und konkav für $\bar{V} \geq 0$. Zusätzlich ist $g_{T, \alpha}(\bar{V})$ stetig auf diesem Intervall, da $g_{T, \alpha}^{-1}(\mu)$ strikt monoton wachsend und stetig für $\mu \geq-12$ ist.

Beide Ansätze unterscheiden sich in der Berücksichtigung der Performance des laufenden Produktes. In Gl. 5 beinhaltet das Chancen- und das Risikomaß sowohl die vergangene Performance des laufenden Produktes als auch die zukünftige des Portfolios, während in Gl. 6 die vergangene Performance bei der Berechnung der Maße keine Rolle spielt. Entscheidend für die Einstufung ist allein die zukünftige Performance des Portfolios. Bei einer gleichen Performance beider Vorsorgeprodukte über die gesamte Vertragslaufzeit unterscheiden sich das Chancen- und das Risikomaß bei beiden Ansätzen nur minimal. Angenommen, das laufende Produkt weist in den ersten $m$ Monaten eine gute Performance auf, die zu einem sehr hohen Vertragsvermögen zum Zeitpunkt $m$ führt, und erzielt danach nur noch eine mäBige Rendite. In Gl. 6 dominiert die zukünftige Performance die Performance der vergangenen $m$ Monate, was zu einem niedrigen Maß führt, obwohl die Gesamtperformance des laufenden Produktes und damit des Portfolios gut ist. Umgekehrt kann trotz einer schlechten Performance in den ersten $m$ Monaten das Maß von Gl. 6 groß sein, wenn die zukünftige Performance gut ist. In beiden Fällen führt Gl. $5 \mathrm{zu}$ einem Maß, das die Gesamtperformance der einzelnen Altersvorsorgeprodukte und des Portfolios besser widerspiegelt, indem unterschiedliche Szenarioverläufe in der Vergangenheit einen geringeren Einfluss haben.

Je nach Art des Altersvorsorgeproduktes wird dem Kunden ein Teil oder das gesamte Vertragsvermögen zum Zeitpunkt $m$ unwiderruflich zugeteilt. Aus dieser Perspektive ist die zukünftige Rendite des Portfolios für den Kunden von Interesse. Dies spricht für eine Klassifizierung des Portfolios gemäß Gl. 6. Wie wir bereits oben diskutiert haben, kann dieser Ansatz jedoch die Performance des laufenden Produktes falsch darstellen. Das Maß, das nach diesem Ansatz berechnet wurde, kann kleiner als die über die gesamte Ansparphase gemittelte jährliche Performance des Produktes sein und umgekehrt. Die Maßdefinition in Gl. 5 berücksichtigt Schwankungen weniger und gibt die Performance des Portfolios über die gesamte Ansparphase korrekt wieder. Diese Argumente sprechen für die Wahl der Maßdefinition nach Gl. 5. Zudem kann der Kunde nicht vollständig über sein aktuelles Vertragsvermögen verfügen, da er bei Kündigung eine Stornogebühr bezahlen muss. Folglich kann die bis zu diesem Zeitpunkt erwirtschaftete Rendite nicht vollständig realisiert werden. Da weiterhin die Produkte gewöhnlich zur Finanzierung der Rentenphase gekauft werden, werden sie in der Regel bis zum Beginn der Rente gehalten. Daher ist die Rendite über die gesamte Ansparphase für den Kunden ausschlaggebend. Aufgrund dieser Argumente empfehlen wir die Berechnung des Chancen- und des Risikomaßes nach Gl. 5 als Rendite über die gesamte Vertragslaufzeit, während die Berechnung nach Gl. 6 nur die Rendite über die verbleibende Vertragslaufzeit berücksichtigt. Trotzdem erscheint hier eine Aussage über den zu verwendenden Ansatz von Seiten des Gesetzgebers erforderlich.

Basierend auf $\mu^{c}$ und $\mu^{r}$ wird die CRK des Portfolios $\alpha V^{\text {lfd }}+(1-\alpha) V^{\text {neu }}$, die sich aus der Interpolation der gemittelten Endvermögen ergibt, bezeichnet mit $\mathscr{C} \mathscr{R} \mathscr{K}{ }^{p t f(\alpha)}$, bestimmt. Hierbei sind die aktuellen CRK-Grenzen der Ansparphase $T^{\text {иеи }}$ zu verwen- 
den. Weiterhin sind die qualitativen Kriterien von CRK 1 und 2 zu berücksichtigen. Gemäß Theorem 1 ist die CRK des Portfolios, $C R K^{p t f(\alpha)}$, nicht größer als $\mathscr{C} \mathscr{R} \mathscr{K}^{p t f(\alpha)}$.

Empfehlungen für die Kundenberatung 3 Wie in Abschn. 3.2, wird die CRK des Portfolios durch Interpolation der gemittelten Endvermögen nach oben abgeschätzt. Für das neue Altersvorsorgeprodukt können die gemittelten Endvermögen der Klassifizierung genutzt werden, sofern sie bekannt sind. Die gemittelten Endvermögen des laufenden Produktes sind zu simulieren. Zur Berechnung des Chancen- und des Risikomaßes aus den gemittelten Endvermögen ist eine modifizierte Funktion zu verwenden, für deren Form wir zwei mögliche Varianten vorgeschlagen haben. Hier sollte eine Empfehlung des Gesetzgebers für die entsprechende Verwendung gemacht werden.

Als Beispiel betrachten wir eine klassische Kapitallebensversicherung, die vor vier Jahren $(m=48)$ mit einem vereinbarten Monatsbeitrag von $100 €$ und einer Vertragslaufzeit von 32 Jahren abgeschlossen wurde. Sie besitzt eine Garantieverzinsung von 1,25\% und der aktuelle Vertragswert beträgt 3931,50€. Zusätzlich wird ein Fondssparplan mit einem monatlichen Beitrag von $200 €$ gekauft, der in einen reinen Aktienfonds mit einer Volatilität von $25 \%$ investiert. Die vereinbarte Vertragslaufzeit beträgt 28 Jahre und entspricht der restlichen Ansparphase der laufenden Lebensversicherung. Der Klassifizierung liegen $\bar{V}^{c \text {, neu }}=126.958,29 €$ und $\bar{V}^{r \text {,neu }}=36.000 €$ zugrunde. Das laufende Produkt besitzt eine vertraglich vereinbarte Bruttobeitragsgarantie und ein stetig wachsendes Kapital, im Gegensatz zum Fondssparplan. Beide Produkte haben die gleichen Vertragskosten: Abschlusskosten in Höhe von 2,5\% der Beitragssumme, die während der ersten fünf Jahre monatlich entnommen werden, und monatliche Verwaltungskosten in Höhe von 7\% des Beitrages. Der Fonds des Fondssparplans hat zusätzlich laufende Kosten in Höhe von $0,3 \%$ des Fondsvermögens.

Zunächst müssen die aktuellen gemittelten Endvermögen der klassischen Lebensversicherung berechnet werden. Auf Basis des skalierten aktuellen Vertragswerts, der aufgrund des vereinbarten Beitrags dem aktuellen Vertragswert $P_{30}$ entspricht, simulieren wir die klassische Lebensversicherung über 30 Jahre mit den aktuellen Marktmodellparametern gemäß PIA, den aktuellen Parametern des Deckungsstocks, einem Garantiezins von 1,25\% und den aktuell gültigen Vertragskosten. Hierbei sind noch Abschlusskosten für ein Jahr zu berücksichtigen. Grundlage dieser Kosten ist die Beitragsumme, die in der Simulation $40.800 €$ beträgt. Wir erhalten $\bar{V}_{48}^{c, \text { lfd }}=58.432,80 €$ und $\bar{V}_{48}^{r \text { lfd }}=52.231,78 €$.

Gemäß den einzelnen Beiträgen ist ein Drittel des Gesamtbeitrages in das laufende Produkt investiert $\left(\alpha=\frac{1}{3}\right)$. Die interpolierten gemittelten Endvermögen des Portfolios sind dann $\bar{V}^{c, \text { int(1/3) }}=104.116,46 €$ und $\bar{V}^{r \text {,int(1/3) }}=41.410,59 €$. Mit Gl. 5 als Funktion zur Berechnung des Chancen- und des Risikomaßes erhalten wir $\mu^{c}=5,65 \%$ sowie $\mu^{r}=0,60 \%$ und mit Gl. $6 \mu^{c}=5,78 \%$ sowie $\mu^{r}=0,66 \%$. In beiden Fällen beträgt die CRK, die sich aus der Interpolation der gemittelten Endvermögen ergibt, 3. Folglich ist die CRK des Portfolios nicht größer als CRK 3. 


\subsubsection{Wahl des Anteils $\alpha$}

Angenommen die oben berechnete CRK des Portfolios als Obergrenze passt nicht mit der Risikopräferenz des Kunden zusammen, d.h. $C R K^{K d}<\mathcal{C R} K^{\mathrm{ptf}(\alpha)}$, so sollte der Kunde nicht den gesamten Betrag $I$ in das neue Produkt investieren. Daher untersuchen wir in diesem Abschnitt, wie die Prämie des neuen Produktes bzw. der Anteil $\alpha$ zu wählen ist, so dass der Kunde kein höheres Risiko als bevorzugt eingeht. Dazu betrachten wir folgende Fälle:

(i) Der Beitrag des laufenden Altersvorsorgeproduktes kann erhöht werden.

(ii) Der Beitrag des laufenden Altersvorsorgeproduktes kann nicht erhöht werden.

Zu Fall (i): Der Beitrag des laufenden Produktes kann erhöht werden.

Als Erhöhung des laufenden Produktes wird entweder die Aufstockung gemäß Vertragsbedingungen oder der Nachkauf des Vertrages zu den gleichen Bedigungen gesehen. Beide Varianten können unterschiedliche Kosten verursachen, die berücksichtigt werden. Wir behandeln die Erhöhung als ein separates Produkt. Somit besteht das Portfolio, das wir betrachten, aus drei Altersvorsorgeprodukten: dem laufenden, der Erhöhung des laufenden Produktes und dem komplett neuen Produkt eines u. U. anderen Typs. Der neue, zu investierende Beitrag $I$ wird in die beiden neuen Produkte aufgeteilt. Der Anteil $\beta$ von $I, 0 \leq \beta \leq 1$, wird für die Aufstockung des laufenden Produktes verwendet. Der verbleibende Anteil $(1-\beta)$ wird in das komplett neue Produkt investiert. Bezogen auf den investierten Gesamtbeitrag ergeben sich folgende Anteile:

- in das laufende Produkt, wie bisher: $\alpha_{\mathrm{lfd}}=\frac{P^{\mathrm{lfd}}}{P^{\mathrm{ffd}}+I}$,

- zur Erhöhung des laufenden Produktes: $\alpha_{\mathrm{erh}}=\frac{\beta I}{P^{\text {lfd }}+I}$,

- in das neue Produkt: $\alpha_{\text {neu }}=\frac{(1-\beta) I}{P^{\text {lfd }}+I}=1-\alpha_{\mathrm{lfd}}-\alpha_{\text {erh }}$.

Zur Berechnung der CRK des Portfolios aus diesen drei Produkten werden die gemittelten Endvermögen benötigt. Neben den aktuellen des laufenden Produktes, $\bar{V}_{m}^{\text {lfd }}$, und denen des komplett neuen, $\bar{V}^{\text {neu }}$, die bekannt sind, werden die gemittelten Endvermögen der Erhöhung, bezeichnet mit $\bar{V}_{0}^{c \text {,erh }}$ und $\bar{V}_{0}^{r \text {,erh }}$, benötigt. Hier sind die anfallenden Kosten zu berücksichtigen. Wir spezifizieren die gemittelten Endvermögen der Erhöhung mit einem Subskript von 0, um zu verdeutlichen, dass diese nicht unbedingt mit den Werten der Klassifizierung übereinstimmen. Wenn das gleiche Produkt zu denselben Bedingungen nochmals gekauft wird, sind die gemittelten Endvermögen der Klassifizierung zu verwenden: $\bar{V}_{0}^{c \text {, erh }}=\bar{V}^{c \text {, lfd }}$ sowie $\bar{V}_{0}^{r \text {,erh }}=\bar{V}^{r \text {,lfd }}$. Wir nehmen an, dass diese bekannt sind. Wenn die Erhöhung gemäß den Vertragsbedingungen dieselben Kosten besitzt wie der Neukauf des Produktes und diese auch zu den gleichen Zeitpunkten entnommen werden, dann sind auch die gemittelten Endvermögen der Klassifizierung zu verwenden. Zusätzlich zu den Kosten müssen auch alle anderen Produktparameter übereinstimmen. Wenn das Produkt nicht mehr verkauft wird, müssen die gemittelten Endvermögen gemäß Abschn. 2.1 simuliert werden. Dasselbe gilt, wenn sich die Kosten oder andere Vertragsparameter bei Erhöhung des laufenden Vertrages gemäß den Vertragsbedingungen von denen eines Neukaufes unterscheiden. Hier sind die entsprechenden 
Kosten sowie Vertragsparameter der Erhöhung in der Simulation zu verwenden. Die gemittelten Endvermögen sind anschließend wie folgt zu interpolieren:

$$
\begin{aligned}
& \bar{V}^{c, \text { int }\left(\alpha_{\mathrm{lfd}}, \alpha_{\mathrm{erh}}\right)}=\alpha_{\mathrm{lfd}} \bar{V}_{m}^{c, \text { lfd }}+\alpha_{\mathrm{erh}} \bar{V}_{0}^{c, \text { erh }}+\alpha_{\text {neu }} \bar{V}^{c, \text { neu }}, \\
& \bar{V}^{r, \text { int }\left(\alpha_{\mathrm{lfd}}, \alpha_{\mathrm{erh}}\right)}=\alpha_{\mathrm{lfd}} \bar{V}_{m}^{r, \text { lfd }}+\alpha_{\mathrm{erh}} \bar{V}_{0}^{r, \text { erh }}+\alpha_{\text {neu }} \bar{V}^{r, \text { neu }} .
\end{aligned}
$$

Wir erweitern Theorem 2 für den Fall eines Portfolios bestehend aus drei Produkten, wobei ein Anteil des Gesamtbeitrags in einem Produkt konstant ist.

Theorem 3 Es sei $g: R_{0}^{+} \rightarrow D$, wobei $D \subseteq \mathbb{R}$, eine streng monoton wachsende Funktion zur Berechnung des Chancen- und des Risikomaßes aus den gemittelten Endvermögen. Weiter sei $V^{1} \in V_{T}$ das Endvermögen eines bereits $m$ Monate laufenden Altersvorsorgeproduktes mit $\bar{V}_{m}^{c, 1}$ und $\bar{V}_{m}^{r, 1}$, die wie in Abschn. 3.3.1 über eine Simulationsphase von $T$ Jahren berechnet sind. Sei $\alpha_{1}$ der konstante Anteil des Gesamtbeitrags, der in $V^{1}$ investiert ist. Seien $V^{i} \in V_{T}, i=2,3$, mit $\bar{V}^{c, i}, \bar{V}^{r, i}$ und $\alpha_{i}$ der Anteil des Gesamtbeitrages, der in $V^{i}$ investiert ist, mit $0 \leq \alpha_{i} \leq\left(1-\alpha_{1}\right)$ und $\alpha_{2}+\alpha_{3}=1-\alpha_{1}$. Es seien weiter $\bar{V}^{c, 2}<\bar{V}^{c, 3}$ und $\bar{V}^{r, 2}>\bar{V}^{r, 3}$, so dass $\mathcal{C} \mathcal{R} K^{\mathrm{ptf}\left(\alpha_{1}, 1-\alpha_{1}\right)} \leq \mathcal{C} \mathcal{R} K^{\mathrm{ptf}\left(\alpha_{1}, 0\right)}$, wobei $\mathcal{C} \mathcal{R} K^{\mathrm{ptf}\left(\alpha_{1}, \alpha_{2}\right)}$ die geschätzte $\mathscr{C} \mathscr{R} \mathscr{K}$ des Portfolios $\alpha_{1} V^{1}+\alpha_{2} V^{2}+\left(1-\alpha_{1}-\alpha_{2}\right) V^{3}$ ist. Schließlich sei $C R K^{K d}$ ein gegebenes Risikoprofil mit $\mathcal{C} \mathcal{R} K^{\mathrm{ptf}\left(\alpha_{1}, 1-\alpha_{1}\right)} \leq C R K^{K d} \leq \mathcal{C} \mathcal{R} K^{\mathrm{ptf}\left(\alpha_{1}, 0\right)}$ und $b_{T}^{C R K^{R d}} \operatorname{der} \mu^{c_{-}}$ Achsenabschnitt der aktuellen CRK-Grenze zwischen $C R K^{K d}$ und $C R K^{K d}+1$ für eine Ansparphase von T. $u\left(\alpha_{1}, \alpha_{2}\right)$ sei definiert als

$$
\begin{aligned}
u\left(\alpha_{1}, \alpha_{2}\right):= & g\left(\alpha_{1} \bar{V}_{m}^{c, 1}+\alpha_{2} \bar{V}^{c, 2}+\left(1-\alpha_{1}-\alpha_{2}\right) \bar{V}^{c, 3}\right) \\
& -g\left(\alpha_{1} \bar{V}_{m}^{r, 1}+\alpha_{2} \bar{V}^{r, 2}+\left(1-\alpha_{1}-\alpha_{2}\right) \bar{V}^{r, 3}\right)
\end{aligned}
$$

und $\alpha_{2}^{*}$ so gewählt, dass es folgende Gleichung löst:

$$
u\left(\alpha_{1}, \alpha_{2}^{*}\right)= \begin{cases}u\left(\alpha_{1}, 0\right) & C R K^{K d}=\mathcal{C} \mathcal{R} K^{\alpha_{1}, 0} \\ b_{T}^{C R K^{K d}} & \text { sonst. }\end{cases}
$$

Dann ist die CRK des Portfolios $\alpha_{1} V^{1}+\alpha_{2} V^{2}+\left(1-\alpha_{1}-\alpha_{2}\right) V^{3}$ nicht größer als $C R K^{K d}$ für $\alpha_{2}>\alpha_{2}^{*}$.

Beweis Analog zum Beweis von Theorem 2 nutzen wir die Abschätzung

$$
\mu^{c, \operatorname{ptf}\left(\alpha_{1}, \alpha_{2}\right)}-\mu^{r, \operatorname{ptf}\left(\alpha_{1}, \alpha_{2}\right)} \leq g\left(\bar{V}^{c, \operatorname{int}\left(\alpha_{1}, \alpha_{2}\right)}\right)-g\left(\bar{V}^{r, \operatorname{int}\left(\alpha_{1}, \alpha_{2}\right)}\right)=u\left(\alpha_{1}, \alpha_{2}\right) .
$$

Da $\alpha_{1}$ konstant ist, hängt $u$ nur von $\alpha_{2}$ ab. Analog zum Beweis von Theorem 2 wird gezeigt, dass $u\left(\alpha_{1}, \alpha_{2}\right)$ streng monoton fallend in $\alpha_{2}$ für $\bar{V}^{c, 2}<\bar{V}^{c, 3}$ und $\bar{V}^{r, 2}>\bar{V}^{r, 3}$ ist und dass genau ein $\alpha_{2}^{*}$ existiert, das die Gl. 7 löst. Für $\alpha_{2}>\alpha_{2}^{*}$ gilt dann

$$
\mu^{r, \operatorname{ptf}\left(\alpha_{1}, \alpha_{2}\right)} \leq u\left(\alpha_{1}, \alpha_{2}\right)<u\left(\alpha_{1}, \alpha_{2}^{*}\right)
$$


Folglich ist in diesem Fall die CRK des Portfolios $\alpha_{1} V^{1}+\alpha_{2} V^{2}+\left(1-\alpha_{1}-\alpha_{2}\right) V^{3}$ nicht größer als $C R K^{K d}$ für $\alpha_{2}>\alpha_{2}^{*}$

Das neue Produkt ist so gewählt, dass es die Anforderungen von Theorem 3 erfüllt. Als Funktion $g$ wird die Umkehrfunktion von Gl. 5 bzw. 6 mit $T^{\text {heu }}$ als $T$ und $\alpha_{l f d}$ als $\alpha$ genutzt. Anwenden von Theorem 3 liefert $\alpha_{\text {erh }}^{*}$ und für $\alpha_{\text {erh }}>\alpha_{\text {erh }}^{*}$ ist die CRK des Portfolios nicht größer als $C R K^{K d}$. Der entsprechende Anteil $\beta^{*}$ von $I$ ergibt sich als

$$
\beta^{*}=\alpha_{\mathrm{erh}}^{*} \frac{\left(P^{\mathrm{lfd}}+I\right)}{I} .
$$

Der maximale Beitrag $P^{*}$ in das neue Produkt, der sicherstellt, dass die CRK des Portfolios nicht größer als $C R K^{K d}$ ist, ist dann $\left(1-\beta^{*}\right) I$.

Empfehlungen für die Kundenberatung 4 Im Fall, dass das laufende Altersvorsorgeprodukt erhöht werden kann, lässt sich der maximal zu investierende Beitrag in das neue Produkt bestimmen, so dass das Portfolio keine höhere CRK als $C R K^{K d}$ besitzt. Dafür muss neben den aktuellen gemittelten Endvermögen des laufenden Produktes ggf. die der Erhöhung simuliert und berechnet werden. Das Vorliegen der qualitativen Kriterien für CRK 1 und 2 ist dabei zu berücksichtigen.

Wir betrachten wieder das obige Beispiel. Die laufende klassische Lebensversicherung kann jetzt erhöht werden. Es entstehen dieselben Kosten wie beim laufenden Produkt und die Abschlusskosten werden ebenfalls über die ersten 60 Monate entnommen. Die Erhöhung besitzt die gemittelten Endvermögen von $\bar{V}_{0}^{c \text {,erh }}=48.379,26 €$ und $\bar{V}_{0}^{r \text {,erh }}=43.655,14$ Euro. Diese sind die Ergebnisse einer Simulation über 30 Jahre basierend auf den aktuellen Marktmodellparametern gemäß PIA, den aktuellen Deckungsstockparametern, einem Garantiezinssatz von $1,25 \%$ und den gültigen Vertragskosten.

Wenn der neue Beitrag von $200 €$ komplett in den Fondssparplan investiert wird, erhalten wir die geschätzte CRK des letzten Abschnitts für beide Funktion von $g_{30,1 / 3}(\bar{V}): \mathcal{C} \mathcal{R} K^{\text {ptf( }(1 / 3,0)}=3$. Ein vollständiges Investment der $200 €$ in die Erhöhung des laufenden Produktes liefert $\mathcal{C} \mathcal{R} K^{\text {ptt( }(1 / 3,2 / 3)}=1$ wiederum für beide Funktion von $g_{30,1 / 3}(\bar{V})$ und den aktuellen CRK-Grenzen von 30 Jahren. Somit sind die Bedingungen $\bar{V}^{c \text {,erh }}<\bar{V}^{c \text {, neu }}, \bar{V}^{r \text { erh }}>\bar{V}^{r \text {, neu }}$ und $\mathcal{C} \mathcal{R} K^{\text {ptf(1/3,2/3) }} \leq$ $\mathcal{C} \mathcal{R} K^{\operatorname{ptf}(1 / 3,0)}$ von Theorem 3 erfüllt. Wir erhalten die in Tab. 2 aufgelisteten Werte $\alpha_{\text {erh }}^{*}$ in Prozent und $\alpha_{\text {neu }}^{*}$ in Prozent für verschiedene $C R K^{K d}$. Das Portfolio kann CRK 1 und 2 aufgrund der fehlenden qualitativen Kriterien nicht erreichen. Trotzdem berechnen wir zur Veranschaulichung auch die Werte für $C R K^{K d}$ von 1 und 2. Ein Beitrag in den Fondssparplan, der geringer als $300 \cdot \alpha_{\text {neu }}^{*}$ ist, stellt sicher, dass die CRK des Portfolios nicht größer als die zugehörige $C R K^{K d}$ ist. Der verbleibende Beitrag $300 \cdot \alpha_{\text {erh }}^{*}$ wird zur Erhöhung des laufenden Vertrages genutzt.

Für verschiedene $\beta \in[0 ; 1]$ werden sowohl $\left(\mu^{c, \text { ptf( }\left(1 / 3, \alpha_{\text {erh }}\right)}, \mu^{r, \operatorname{ptf}\left(1 / 3, \alpha_{\text {ert }}\right)}\right)$ des Portfolios als auch $\left(\mu^{c}, \mu^{r}\right)$ der interpolierten gemittelten Endvermögen $\bar{V}^{c \text {,int }\left(1 / 3, \alpha_{\text {erh }}\right)}$ und $\bar{V}^{r \text {,int }\left(1 / 3, \alpha_{\text {ert }}\right)}$ berechnet. Diese Parameter und die zugehörige CRK sind in Abb. 7 für beide Funktionen von $g_{30,1 / 3}(\bar{V})$ veranschaulicht. Genau wie in Abb. 4 repräsentieren die untere gestrichelte Linie das Chancen- und das Risikomaß der inter- 
polierten gemittelten Endvermögen und die obere Linie die tatsächlichen Maße des Portfolios. Jeder Punkt auf der Linie gehört zu einem $\beta$. Die gleichen $\beta$ auf beiden Linien sind durch einen vertikalen Pfeil verbunden, der die Richtung spezifiziert, in die die interpolierten Werte zu verschieben sind, um die tatsächlichen zu erhalten. $\beta$ geht von $1 \mathrm{zu} 0$ vom Punkt in der linken oberen Ecke zu dem rechten unteren. Für $\beta=1$ sind die tatsächlichen Maße und die der interpolierten gemittelten Endvermögen nicht die gleichen, auch wenn es in der Abbildung so erscheint. Die Differenz hier ist sehr gering. Genauso wie für $\beta=0$ wird hier immer ein Teil der Gesamtprämie im laufenden Altersvorsorgeprodukt investiert. Die Szenarioverteilung der Endvermögen des laufenden Produktes unterscheidet sich zu der der Erhöhung, auch wenn beide Produkte ein und dasselbe Produkt mit unterschiedlichen Laufzeiten sind. Somit ist die Interpolation der gemittelten Endvermögen für $\beta=1$ genauso wie für $\beta=0$ eine Abschätzung des Portfolios. Die Punktform gibt an, in welchem Intervall $\beta$ abhängig von $\beta_{j}^{*}$ liegt. Das Intervall, in dem $\beta$ in Bezug auf $\beta_{j}^{*}$ liegt, ist in der Abbildung durch die verschiedene Punktarten Kreis, Dreieck und Viereck dargestellt. Wir bezeichnen mit $\beta_{j}^{*}$ das $\beta^{*}$, das zu $C R K^{K d}=j, j=1, \ldots, 3$, gehört. Die Aussage von Theorem 3 ist deutlich zu erkennen. Für $\beta>\beta_{j}^{*}$ ist die CRK des Portfolios nicht größer als die CRK, die man durch Interpolation der gemittelten Endvermögen erhält, die wiederum nicht größer als CRK $j$ ist.

Zu Fall (ii): Der Beitrag des laufenden Produktes kann aufgrund der Vertragsbedingungen nicht erhöht werden.

In diesem Fall kann der Beitrag zum laufenden Altersvorsorgeprodukt nicht erhöht werden, weil dies entweder die Vertragsbedingungen ausschließen oder das Produkt nicht mehr zu den gleichen Bedingungen verkauft wird. Der Kunde kann in diesem Fall nur entscheiden, wie hoch die Prämie sein soll, die in das neue Produkt investiert wird. Dies ist die einzige Größe, über die die CRK des Portfolios gesteuert werden kann. Wenn bei Investition des gesamten Betrages $I$ in das neue Produkt die CRK des Portfolios höher als die Risikopräferenz des Kunden ist, so kann die CRK des Portfolios nur durch eine niedrigere Prämienzahlung in das neue Produkt als $I$ verringert werden. Das bedeutet, dass nicht der gesamte Betrag $I$ investiert wird. In diesem Fall sind der Beitrag des neuen Produktes, $P^{\text {neu }}$, und damit $\alpha$ anzupassen bis die gewünschte CRK aus der Berechnung in Abschn. 3.3.1 resultiert. Für CRK 1 bzw. 2 als $C R K^{K d}$ müssen sowohl das laufende als auch neue Produkt die zugehörigen qualitativen Kriterien erfüllen.

Empfehlungen für die Kundenberatung 5 Für jeden möglichen Beitrag in das neue Altersvorsorgeprodukt ist die geschätzte CRK des Portfolios nach Abschn. 3.3.1 zu berechnen. Dabei kann man mit dem maximal verfügbaren Beitrag beginnen, der für das neue Produkt zur Verfügung steht, und ihn solange stückweise senken, bis die gewünschte $C R K^{K d}$ erreicht ist. Der Beitrag im laufenden Produkt bleibt gleich. Die qualitativen Kriterien von CRK 1 und 2 sind zu berücksichtigen.

Wir betrachten den Fall, dass in unserem Beispiel die klassische Lebensversicherung nicht erhöht werden kann. Für verschiedene Beiträge mit $P^{\text {neu }} \in[0 ; 200]$ in den Fondssparplan wird die geschätzte CRK des Portfolios wie in Abschn. 3.3.1 beschrieben berechnet. Wir erhalten den maximal in den Fondssparplan zu investierenden Beitrag $P^{*}$ in $€$ für verschiedene $C R K^{K d}$, der in Tab. 3 zu finden ist. Wenn 
Tab. $2 \alpha_{l f d}, \alpha_{\mathrm{erh}}^{*}$ sowie $\alpha_{\text {neu }}^{*}$ abhängig von $C R K^{K d}$ eines Portfolios aus einem laufenden und neuem Produkt unter der Möglichkeit der Erhöhung des laufenden Produktes

\begin{tabular}{lllll}
\hline & $g_{T}$ & \multicolumn{1}{c}{$C K^{K d}$} & \\
& & 1 & 2 & 3 \\
\hline$\alpha_{\text {lfd }}$ in \% & & 33,33 & 33,33 & 33,33 \\
$\alpha_{\text {erh }}^{*}$ in \% & Gl. 5 & 55,40 & 33,40 & 0 \\
& Gl. 6 & 55,66 & 34,09 & 0 \\
$\alpha_{\text {neu }}^{*}$ in \% & Gl. 5 & 11,26 & 33,26 & 66,66 \\
& Gl. 6 & 11,01 & 32,58 & 66,66 \\
\hline
\end{tabular}

der Kunde ein Risikoprofil von 2 besitzt, so sollten maximal 59,11 bzw. 56,26€ in den Fondssparplan investiert werden, damit das Portfolio keine größere CRK als 2 hat. Weiterhin werden $100 €$ monatlich in die klassische Lebensversicherung eingezahlt.

\section{Zusammenfassung und Empfehlungen}

Die Klassifizierung staatlich geförderter Altersvorsorgeprodukte durch die PIA stellt ein wichtiges Werkzeug des Verbraucherschutzes dar, da sie es dem Kunden ermöglicht, ein solches Produkt im Hinblick auf sein Verhältnis von Chancen und Risiko zu beurteilen. Gemeinsam mit dem vom EI-QFM entwickelten Verfahren zur Ermittlung der Chancen-Risiko-Neigung des Kunden stehen dem Berater im Kundengespräch zwei nützliche Werkzeuge zur Verfügung, um eine optimale, individuell auf den Kunden ausgerichtete Beratung im Hinblick auf passende Altersvorsorgeprodukte durchzuführen.

Um allerdings einen Kunden passend zu beraten, der bereits Altersvorsorgeprodukte besitzt, ist es notwendig, eine CRK des sich ergebenden Portfolios aus bereits vorhandenem und neu zu erwerbendem Produkt zu ermitteln. Hierzu haben wir im Rahmen dieses Artikels eine geeignete Vorgehensweise entwickelt. Ihre Basis stellt die Untersuchung der Eigenschaften der verschiedenen Chancen- und Risikoparameter dar. Dabei ist nur die Abbildung auf den Mittelwert der Endvermögen, also das Vertragsvermögen am Ende der Ansparphase, linear in der Kombination zweier Produkte, während die anderen Maße Konkavität aufweisen. Dabei machen wir die - zumindest approximativ gerechtfertigte - Annahme der Linearität der Kosten der Produkte im Endvermögen (siehe hierzu auch Bemerkung 1). Aufbauend auf den Eigenschaften haben wir gezeigt, dass ein Diversifikationseffekt bei der Klassifizierung gemäß PIA vorliegt, es also z.B. möglich ist, durch Kombination von Produkten auch Produkte einer höheren CRK, als der des Kunden, in das Portfolio einzubeziehen und trotzdem insgesamt die gewünschte CRK zu erhalten, dabei aber z.B. die Renditechancen zu erhöhen.

Ein weiteres wichtiges Ergebnis unsere Analyse ist, dass für eine Abschätzung der CRK des Portfolios nach oben nach Theorem 1 die gemittelten Endvermögen $\bar{V}^{c}$ und $\bar{V}^{r}$ zu verwenden sind. Diese sind gemäß ihren Anteilen zu interpolieren und die sich dann daraus ergebenden Chancen- und Risikomaße zu berechnen. Im 


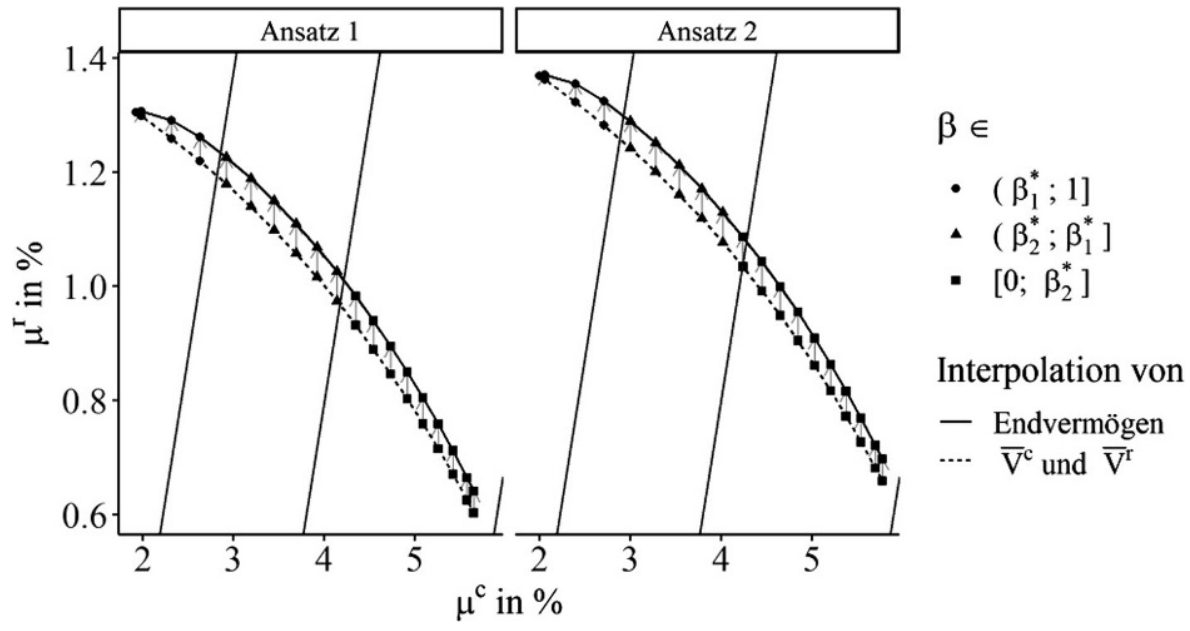

Abb. 7 Veranschaulichung der Beziehung zwischen $\beta^{*}, \mathcal{C} \mathcal{R} K^{\mathrm{ptf}\left(1 / 3, \alpha_{\mathrm{erh}}\right)}$ und der CRK des Portfolios

Fall eines Portfolios aus zwei neuen Altersvorsorgeprodukten können die Anteile an den einzelnen Produkten bestimmt werden, so dass die CRK des Portfolios aus beiden Produkten nicht größer als eine vorgegebene CRK ist. Unsere Diversifikationsergebnisse konnten wir auch auf den Fall eines Portfolios aus einem bereits laufenden und einem neuen Produkt übertragen, wobei es möglich sein muss den Beitrag des laufenden Produktes zu erhöhen. Ist dies nicht der Fall, so muss für jeden Anteil am neuen Produkt die Obergrenze der CRK des Portfolios bestimmt werden, bis sie die gewünschte CRK aufweist. Hier ist der Zukauf des neuen Produkts durch das Erreichen der gewünschten CRK begrenzt. Der Zukauf erfolgt schrittweise.

Hieraus ergibt sich die Notwendigkeit für den Gesetzgeber, entsprechende Regelungen zu schaffen, wie der Berater diese Endvermögen im Kundengespräch verwenden kann, ohne sie explizit zu kennen, da nach bisheriger Regelung weder die expliziten Werte des Chancen- und des Risikomaßes noch die von der PIA verwendeten simulierten Endvermögen der Produkte vom Anbieter extern kommuniziert werden dürfen. Nur so sind die von uns aus unseren theoretischen Resultaten hergeleiteten Empfehlungen für die Kundenberatung tatsächlich vom Berater umsetzbar.

Unsere Ergebnisse sind nicht nur für die Beratung von Nutzen, sondern können auch für den Versicherer im Hinblick auf eine kostengünstige und passgenaue Produktentwicklung interessant sein. So ermöglicht es die Bestimmung bzw. Begrenzung der CRK eines Portfolios dem Anbieter, aus seinen vorhandenen Produkten durch geeignete Portfoliobildung für die jeweilige CRK (approximativ) optimale Produkte zu konstruieren, in dem Sinn, dass zu einer vorgegebenen (Kunden-) CRK, die zwischen den CRK zweier vorhandener Produkte liegt, mit Hilfe von Theorem 2 eine Konvexkombination dieser Produkte bestimmt werden kann, die das mit den beiden Produkten höchstmöglich erzielbare Chancenmaß in der vorgegebenen CRK (approximativ im Sinne der Abschätzung der CRK nach oben) erreicht. Hierfür muss der Anbieter keine komplett neuen Produktkonzepte entwickeln. Gleichzeitig könnte der Anbieter auch noch Kostenvorteile bewerben, wenn er sich dazu entschließen 
Tab. 3 Maximal in den Fondssparplan zu investierender Geldbetrag $P^{*}$ abhängig von $C R K^{K d}$ eines Portfolios aus einem laufenden und einem neuem Produkt ohne Möglichkeit der Erhöhung des laufenden Produktes

\begin{tabular}{lllll}
\hline & $g_{T}$ & \multicolumn{2}{l}{ CR $K^{K d}$} & \\
& & 1 & 2 & 3 \\
\hline$P^{*}$ in $€$ & Gl. 5 & 15,81 & 59,11 & 200 \\
& Gl. 6 & 14,99 & 56,26 & 200 \\
\hline
\end{tabular}

könnte, für ein Portfolio lediglich Fixkosten in Höhe eines der beiden beteiligten Produkte zu berechnen, falls beide von ihm erworben werden.

Schließlich können prinzipiell in unsere Analyse auch andere Anlagen des Kunden als staatlich geförderte Altersvorsorgeprodukte einbezogen werden. Dafür sind diese allerdings gemäß dem Modell der PIA zu klassifizieren.

Danksagung Die Autoren bedanken sich bei Daniel Krüger für seine tatkräftige Unterstützung bei der Formatierung des Artikels.

Förderung Franziska Diez wurde durch ein Promotionsstipendium des Fraunhofer ITWM gefördert.

Funding Open Access funding enabled and organized by Projekt DEAL.

Open Access Dieser Artikel wird unter der Creative Commons Namensnennung 4.0 International Lizenz veröffentlicht, welche die Nutzung, Vervielfältigung, Bearbeitung, Verbreitung und Wiedergabe in jeglichem Medium und Format erlaubt, sofern Sie den/die ursprünglichen Autor(en) und die Quelle ordnungsgemäß nennen, einen Link zur Creative Commons Lizenz beifügen und angeben, ob Änderungen vorgenommen wurden.

Die in diesem Artikel enthaltenen Bilder und sonstiges Drittmaterial unterliegen ebenfalls der genannten Creative Commons Lizenz, sofern sich aus der Abbildungslegende nichts anderes ergibt. Sofern das betreffende Material nicht unter der genannten Creative Commons Lizenz steht und die betreffende Handlung nicht nach gesetzlichen Vorschriften erlaubt ist, ist für die oben aufgeführten Weiterverwendungen des Materials die Einwilligung des jeweiligen Rechteinhabers einzuholen.

Weitere Details zur Lizenz entnehmen Sie bitte der Lizenzinformation auf http://creativecommons.org/ licenses/by/4.0/deed.de.

\section{Literatur}

Acerbi, C., Tasche, D.: On the coherence of expected shortfall. J. Bank. Finance 26, 1487-1503 (2002)

Bundesministerium der Finanzen: Produktinformationsblatt gemäß 7 Altersvorsorgeverträge-Zertifizierungsgesetz (AltZertG); Amtlich vorgeschriebenes Muster gemäß 13 Altersvorsorge-Produktinformationsblattverordnung (AltvPIBV). NWB Verlag, Herne (2019). IV C PIA - S 2220 a/16/10003:004, BStB1 I 2019, S. 240, Schreiben vom 14.03.2019

Glau, K., Linders, D., Min, A., Scherer, M., Schneider, L., Zagst, R.: Innovations in insurance, risk- and asset management. World Scientific Publishing Co. Pte. Ltd., Singapur (2018)

Korn, R., Andelfinger, V.P.: Der Kunde - Chance und Risiko im Beratungsgespräch. Z. Versicherungswes. 17, 538-540 (2016)

Korn, R., Wagner, A.: Chance-risk classification of pension products: Scientific concepts and challenges. In: Glau, K., Linders, D., Min, A., Scherer, M., Schneider, L. (Hrsg.) Innovations in insurance, riskand asset management, S. 381-398. World Scientific Publishing Co. Pte. Ltd., Singapur (2018)

Korn, R., Wagner, A.: Praxishandbuch Lebensversicherungsmathematik: Simulation und Klassifikation von Produkten, 1. Aufl. VVW GmbH, Karlsruhe (2019) 
Produktinformationsstelle Altersvorsorge: Beschreibungstexte für die Chancen-Risiko-Klassen im Produktinformationsblatt (2016). https://produktinformationsstelle.de/wp-content/uploads/2018/08/ CRK-Texte-PIB_082016.pdf, Zugegriffen: 20. Juli 2021 